\title{
Quebra da Simetria Eletrofraca e uma Quarta Geração
}

\author{
Carlos Eduardo Ferreira Haluch
}

Dissertação apresentada ao Instituto de Física da Universidade de São Paulo para a obtenção do título de Mestre em Ciências.

Orientador: Prof. Dr. Gustavo Burdman

Comissão Examinadora:

Prof. Dr. Oscar José Pinto Éboli

Prof. Dr. Fernando Silveira Navarra

Prof. Dr. Alex Gomes Dias

São Paulo 


\title{
FICHA CATALOGRÁFICA
}

\author{
Preparada pelo Serviço de Biblioteca e Informação \\ do Instituto de Física da Universidade de São Paulo
}

\begin{abstract}
Haluch, Carlos Eduardo Ferreira
Quebra da Simetria Eletrofraca e uma Quarta Geração - São Paulo, 2009.

Dissertação (Mestrado) - Universidade de São Paulo. Instituto de Física Depto. de Física Matemática
\end{abstract}

Orientador: Prof. Dr. Gustavo Burdman

Área de Concentração: Física

Unitermos: 1. Física de Partículas; 2. Física Teórica; 3. Física de Alta Energia.

$\mathrm{USP} / \mathrm{IF} / \mathrm{SBI}-085 / 2009$ 


\section{Resumo}

O Modelo Padrão da física de partículas descreve com sucesso as interações eletrofracas e fortes da natureza, pois suas previsões estão de acordo com os dados experimentais. No entanto, esse modelo apresenta alguns problemas, como os problemas da hierarquia e o da massa dos férmions. Uma forma de resolver esses problemas foi proposta por Burdman e Da Rold, que consideraram uma quarta geração de férmions fortemente acoplada na escala $\mathrm{TeV}$ (escala de energias a ser explorada no LHC), como origem da quebra da simetria eletrofraca. Esse modelo, baseado na correspondência AdS/CFT, propõe uma teoria com uma dimensão extra compacta num orbifold $S_{1} / Z_{2}$ (Randall-Sundrum) como descrição dual de uma teoria $4 \mathrm{D}$ fortemente acoplada à baixas energias $(\sim 1 \mathrm{TeV})$. Nesse trabalho nós estudamos a fenomenologia desse modelo para a produção de léptons da quarta geração. Argumentamos que embora as seções de choque para esses processos sejam pequenas, da ordem de $30 \mathrm{fb}$, eles são a melhor alternativa para observar uma quarta geração de férmions acoplada com os modos excitados dos bósons de KK, sendo a observação desse sinal necessária para corroborar esse modelo de quebra da simetria eletrofraca. 


\section{Abstract}

The Standard Model of particle physics successfully describes the electroweak and strong interactions of nature, because their predictions are in agreement with the experimental data. However, this model presents some problems, the problems of hierarchy and the mass of fermions. One way to solve these problems was proposed by Burdman and Da Rold, who considered a fourth generation of fermions strongly coupled to the TeV scale (scale of energy to be explored at the LHC) as the origin of electroweak symmetry breaking. This model, based on the AdS / CFT correspondence, proposed a theory with an compact extra dimension on a orbifold $S_{1} / Z_{2}$ (Randall-Sundrum) as a dual description of a $4 \mathrm{D}$ strongly coupled theory at low energies $(\sim 1 \mathrm{TeV})$. In this paper we study the phenomenology of this model for the production of leptons of the fourth generation. We argue that although the cross sections for these processes are small, of order $30 \mathrm{fb}$, they are the best way to see a fourth generation of fermions coupled with the modes of excited bosons KK, and the observation of this signal required to support this model electroweak symmetry breaking. 


\section{Agradecimentos}

Quero agradecer inicialmente a Deus, que entre muitas escolhas na minha vida me levou a esse trabalho, deixando de lado as paixões iniciais pela teologia, história e filosofia. No entanto, foi só por meio delas que pude encontrar a física, em particular o mundo maravilhoso da cosmologia e da física de partículas.

Quero agradecer também ao meu professor e orientador Gustavo Burdman, que desde o início desse trabalho me acompanhou e ensinou, sempre atenciosamente e com paciência, e mesmo nos momentos mais difíceis me deu motivação para não desistir.

À minha companheira amada Amanda e à minha querida mãe Elisabete meus agradecimentos especiais, por sempre acreditarem em mim, e nos momentos mais difícieis me apoiarem e estarem ao meu lado. Ao meu pai, Carlos Artur, às minhas irmãs Carolina e Ghabriela, aos meus tios Isaías, Magda e Enoy, e a toda minha família meus sinceros agradecimentos pelo apoio e motivação durante todo o trabalho.

Aos meus colegas e amigos, em especial ao Ricardo, Leandro, João, Renann, Juliano, Dorival, entre outros. Eles foram muitos importantes, tanto pela companhia como pela ajuda e apoio durante a execução do trabalho.

Quero agradecer também a um pessoal que sempre me inspirou ou motivou de alguma forma, Jesus, Voltaire, Newton, Kant, Spinoza, Hawking, Platão, Paulo, Sócrates, Arnold, Adorno, Popper, Hobbes, Einstein, Ghandi, etc. 
à minha avó Shirley 
"Sentimos que, mesmo depois de serem respondidas todas as questões científicas possiveis, os problemas da vida permanecem completamente intactos."

\section{Ludwig Wittgenstein.}

vii 


\section{Sumário}

1 Introdução $\quad 3$

2 Modelo Padrão $\quad 6$

2.1 Invariância de Gauge e a QED . . . . . . . . . . . . . . . . 6

2.2 Invariância de Gauge para grupos não-abelianos . . . . . . . . 9

2.3 Quebra espontânea de simetria . . . . . . . . . . 11

2.4 O Mecanismo de Higgs . . . . . . . . . . . . . . . . . . . . 14

2.5 Modelo das Interações Eletrofracas . . . . . . . . . . . . . 18

2.5.1 A estrutura $V-A \ldots \ldots \ldots$

2.5.2 Modelo Eletrofraco . . . . . . . . . . . . . . . . 19

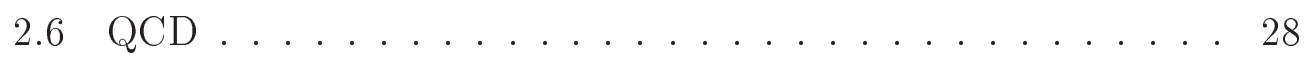

2.6.1 Introduzindo os Quarks . . . . . . . . . . . . 28

2.6.2 Anomalias ................. . 30

2.6.3 Massa dos Quarks e Matriz CKM . . . . . . . . 31

2.7 Modelo Padrão e suas interações . . . . . . . . . . . . . . . . . 34

3 Além do Modelo Padrão 36

3.1 Problemas do MP . . . . . . . . . . . . . . . . . 37

3.1 .1 Problema da Hierarquia . . . . . . . . . . . . 37 
3.1.2 O Problema da Massa dos Férmions . . . . . . . . 41

3.1.3 Outros Problemas . . . . . . . . . . . . . 42

3.2 Teorias além do Modelo Padrão . . . . . . . . . . . . . . . . . 43

3.2.1 Teorias com Dimensões Extras . . . . . . . . . 43

3.2.2 Teorias com Dimensões Extras Curvas (WED) . . . . . 46

4 Quebra da Simetria Eletrofraca $\quad 56$

4.1 Condensação do Quark e Quebra da Simetria Eletrofraca . . . 57

4.2 O Modelo . . . . . . . . . . . . . . . . . . 62

4.2.1 Acoplamentos de KK e Operadores de Dimensão Maior 62

4.2.2 Condensação da quarta geração e EWSB . . . . . . . . 66

4.2.3 Vínculos de precisão eletrofracos . . . . . . . . . . . 71

4.3 Fenomenologia ................... . . 73

4.3.1 Quarks da Quarta Geração . . . . . . . . . . 73

4.3.2 Léptons da Quarta Geração . . . . . . . . . . . . . . 74 


\section{Capítulo 1}

\section{Introdução}

O Modelo Padrão da física de partículas é uma teoria quântica de campos desenvolvida nas décadas de 60 e 70 inicialmente por Glashow, Salam e Weinberg [1, 2]. Esse modelo descreve três, das quatro, interações fundamentais da natureza, as forças forte, fraca e eletromagnética. As partículas fundamentais do modelo dividem-se em duas estatísticas distintas, os férmions que são partículas de spin semi-inteiro obedecem a estatística de Fermi-Dirac; e os bósons, que são partículas de spin inteiro e obedecem a estatística de Bose-Einstein. Os férmions são as partículas que constituem a matéria e os bósons são as partículas de força, que mediam as interações entre os férmions. Os férmions são divididos em quarks e léptons, ambos divididos em três famílias ou gerações. Os quarks são os únicos que sentem a interação forte, mediada pelos glúons, caracterizados pelo número quântico cor, além disso eles também possuem carga elétrica e sabor (número quântico que distingue os diferentes tipos de quark/lépton), podendo sentir as interações eletrofracas, mediadas pelos bósons $W^{ \pm}, Z$ e o fóton. Os léptons não possuem cor e portanto não participam das interações fortes, mas sentem as interações eletrofracas, com exceção dos neutrinos que tem carga nula, só participando das interações fracas. O grupo de simetria do modelo é $S U(3)_{c} \times S U(2)_{L} \times U(1)_{Y}$, especificando que os quarks são tripletos de cor, os férmions de mão esquerda são organizados em dubletos, enquanto os férmions de mão direita são singletos. 
Um dos grandes sucessos do Modelo Padrão está na observação das correntes neutras nos anos 70, assim como a previsão para as massas dos bósons de gauge, observadas na década de 80 , em pleno acordo com a teoria. No entanto, a partícula escalar responsável por dar massa a todas as partículas do modelo, o bóson de Higgs, ainda não foi encontrada. No MP, o bóson de Higgs é a única partícula escalar, que é inclusa no modelo através do mecanismo de quebra espontânea de simetria. Embora essa partícula seja fundamental no modelo, a sua presença cria um problema de naturalidade na teoria, pois às correções radiativas a sua massa possuem divergências quadráticas com o cuttof. Isso cria um problema de hierarquia, se o cutoff da teoria é a escala de Planck $\left(\Lambda \simeq 10^{19} \mathrm{GeV}\right)$, pois o limite teórico para a massa do Higgs deve ser da ordem de $1 \mathrm{TeV}$, necessitando um grande ajuste fino. Outro problema de hierarquia do modelo é relacionado à massa dos férmions, que são 12 parâmetros livres na teoria ajustados experimentalmente. O MP não explica porque partículas com os mesmos números quânticos, com massas geradas da mesma maneira e com o mesmo acoplamento de gauge, possuem massas tão diferentes, variando até 6 ordens de grandeza.

Esses e outros problemas são a motivação para o estudo de teorias físicas além do Modelo Padrão, como supersimetria, technicolor, teorias com dimensões extras, etc. Nesse trabalho estaremos interessados em apresentar as soluções do problema da hierarquia e da massa dos férmions utilizando teorias com dimensões extras curvas e uma teoria fortemente acoplada em 4-dimensões [3]. A idéia é explicar a origem da quebra da simetria eletrofraca através de uma teoria 4D fortemente acoplada na escala $\mathrm{TeV}$, a escala da nova física. Essa teoria por sua vez, pode ser descrita por uma teoria com uma dimensão extra curva fracamente acoplada, de acordo com a correspondência AdS/CFT [4]. Esse modelo [5] propõe uma quarta geração de férmions pesada, sendo que os quarks U dessa família condensam e quebram a simetria eletrofraca. O problema da naturalidade é resolvido e temos um Higgs pesado composto por $\bar{U} U$. A interação de 4-férmions do modelo tem duas contribuições importantes, os acoplamentos com os bósons de Kaluza-Klein, e as interações induzidas por operadores de dimensão maior, que geram as massas de todos os outros férmions do modelo. O objetivo principal dessa dissertação é es- 
tudar a fenomenologia da quarta geração de léptons desse modelo de quebra da simetria eletrofraca [6]. Mostraremos o sinal para a observação da quarta geração de léptons no LHC, os acopalmentos, larguras, seções de choque, e os possíveis backgrounds para esse sinal.

No capítulo 2 apresentamos o Modelo Padrão da física de partículas. De forma gradual introduzimos as ferramentas básicas da teoria quântica de campos necessárias para a construção do modelo, o princípio da invariância de gauge para teorias com simetrias global e local, abelianas e não-abelianas, a quebra espontânea de simetria, o teorema de Goldstone e o mecanismo de Higgs. Em seguida discutimos o modelo eletrofraco e suas previsões, finalizando o capítulo com a QCD as interações do MP.

O capítulo 3 começa discutindo os problemas do Modelo Padrão, com ênfase nos problemas da hierarquia de escalas e da massa dos férmions. Nas seções seguintes apresentamos as teorias além do MP necessárias para a construção do modelo de quebra da simetria eletrofraca por uma quarta geração. O enfoque principal são nas teorias com dimensões extras curvas, modelo de Randall-Sundrum, considerando férmions e bósons de gauge livres para se propagar pelas dimensões extras e o Higgs confinado na brana $\mathrm{TeV}$.

No capítulo 4 discutimos o modelo que estamos estudando [5], apresentando inicialmente o modelo de Nambu-Jona-Lasinio, que explica a quebra dinâmica da simetria eletrofraca através de uma interação de 4-férmions. Nas seções seguintes mostramos os acoplamentos dos férmions do MP e da quarta geração com os bósons de KK, as interações de 4-férmions induzidas por operadores de dimensão maior, o mecanismo de quebra da simetria eletrofraca e os vínculos de precisão eletrofracos desse modelo. Na última seção apresentamos o nosso trabalho, discutindo a fenomenologia da produção de léptons da quarta geração desse modelo. 


\section{Capítulo 2}

\section{Modelo Padrão}

Vamos iniciar esse trabalho apresentando o Modelo Padrão da física de partículas, construído entre as décadas de 60 e 70 inicialmente por Glashow, Salam e Weinberg. O MP descreve as interações eletrofracas e fortes entre as partículas elementares da natureza, os férmions (quarks e léptons), mediadas pelos bósons (fóton, $W^{ \pm} \mathrm{e} Z^{0}$ nas interações eletrofracas; glúons nas interações fortes). Antes de apresentarmos o MP vamos introduzir os principais ingredientes da teoria quântica de campos para a construção do modelo, a invariância de gauge, a quebra espontânea de simetria e o mecanismo de Higgs.

\subsection{Invariância de Gauge e a QED}

Para construir o MP vamos começar com exemplos mais simples, como é o caso de uma teoria sem interações. A Lagrangiana para férmions livres é dada por

$$
\mathcal{L}_{0}=\bar{\psi}(x)(i \not \partial-m) \psi(x),
$$

onde $\psi(x)$ é o campo do férmion de massa $m$, e $\bar{\psi}(x)$ o campo do anti-férmion de mesmo sabor. Essa Lagrangiana é invariante sob a transformação de fase 
global $\psi=e^{i \alpha} \psi$, sendo $\alpha$ uma constante. A invariância da Lagrangiana sob essa transformação implica na conservação da corrente global

$$
\partial_{\mu} j^{\mu}=0
$$

onde

$$
j^{\mu}(x)=\bar{\psi}(x) \gamma^{\mu} \psi(x) .
$$

No entanto a Lagrangiana (2.1) não é invariante sob a transformação de fase local $\psi=e^{i \alpha(x)} \psi$. Podemos tornar a Lagrangiana invariante introduzindo um novo campo, o campo de gauge $A_{\mu}$. Definindo a derivada covariante como

$$
D_{\mu} \equiv \partial_{\mu}+i e A_{\mu}
$$

onde e é a constante de acoplamento (carga elétrica do elétron na QED). A Lagrangiana se torna invariante sob as seguintes tranformações de fase locais:

$$
\begin{gathered}
\partial^{\mu} \rightarrow D^{\mu} \\
\psi(x) \rightarrow \psi^{\prime}(x)=e^{i \alpha(x)} \psi(x) \\
A^{\mu}(x) \rightarrow A^{\prime \mu}(x)=A^{\mu}(x)-\frac{1}{e} \partial^{\mu} \alpha(x) .
\end{gathered}
$$

Substituindo essas transformações na Lagrangiana do campo livre $\psi$, obtemos

$$
\begin{gathered}
\mathcal{L} \rightarrow \mathcal{L}^{\prime}=\bar{\psi}^{\prime}\left[\left(i \not \partial-e A^{\prime}\right)-m\right] \psi^{\prime} \\
=\bar{\psi} e^{-i \alpha}\left[i \not \partial-e\left(A-\frac{1}{e} \not \partial \alpha\right)-m\right] e^{i \alpha} \psi=\mathcal{L}-e \bar{\psi} \gamma_{\mu} A^{\mu} \psi,
\end{gathered}
$$

onde o último termo da Lagrangiana pode ser identificado como um termo de interação entre o bóson de gauge e o férmion. 
Com esse resultado podemos observar que a simetria da Lagrangiana implica em uma nova dinâmica para a teoria, já que a imposição da invariância local necessita da introdução de um novo campo, o campo de gauge $A^{\mu}$, e gera um termo de interação entre esse campo e o campo fermiônico $\psi$. A idéia de gerar termos de interação entre os campos através de simetrias impostas a Lagrangiana foi proposta por Salam e Ward [7].

Para obtermos a Lagrangiana da eletrodinâmica quântica (QED, Quantum Eletrodynamics), uma teoria quântica de campos que descreve todos os fenômenos envolvendo partículas eletricamente carregadas interagindo através da força eletromagnética, precisamos introduzir o termo cinético do campo $A^{\mu}$ (o fóton nessa teoria), que é dado por

$$
\mathcal{L}_{\mathrm{A}}=-\frac{1}{4} F_{\mu \nu} F^{\mu \nu}
$$

onde

$$
F_{\mu \nu}=\partial_{\mu} A_{\nu}-\partial_{\nu} A_{\mu}
$$

O termo de massa do campo de gauge $A_{\mu} A^{\mu}$ não é invariante sob a transformação de fase local, o que implica que o fóton não pode ter massa.

Adicionando o termo cinético (2.7) na equação (2.6), obtemos a Lagrangiana da eletrodinâmica

$$
\begin{gathered}
\mathcal{L}_{\mathrm{QED}}=\mathcal{L}_{\text {férm. }}+\mathcal{L}_{\mathrm{A}}+\mathcal{L}_{\text {int. }}, \\
\mathcal{L}_{\mathrm{QED}}=\bar{\psi}(i \not \partial-m) \psi-\frac{1}{4} F_{\mu \nu} F^{\mu \nu}-e \bar{\psi} \gamma_{\mu} A^{\mu} \psi \\
=\bar{\psi}(i \not D-m) \psi-\frac{1}{4} F_{\mu \nu} F^{\mu \nu}
\end{gathered}
$$




\subsection{Invariância de Gauge para grupos não-abelianos}

Em 1954, Yang e Mills [8] aplicaram o princípio da invariância de gauge para grupos não-abelianos. Considerando os campos

$$
\Psi=\left(\begin{array}{c}
\psi_{1} \\
\psi_{2} \\
\cdot \\
\cdot \\
\cdot \\
\psi_{n}
\end{array}\right)
$$

e um grupo com geradores $t_{a}$, que obedece a álgebra de Lie

$$
\left[t_{a}, t_{b}\right]=i C_{a b c} t_{c}
$$

onde $C_{a b c}$ são as constantes de estrutura do grupo. O campo $\Psi$ se transforma como

$$
\Psi(x) \rightarrow \Psi^{\prime}(x)=U(x) \Psi(x)
$$

onde

$$
U(x) \equiv e^{-i T^{a} \alpha^{a}(x)},
$$

sendo que $T^{a}$ é uma representação de $t^{a}$. Nesse caso, a derivada covariante é definida da seguinte forma

$$
D_{\mu} \equiv \partial_{\mu}-i g T^{a} A_{\mu}^{a}
$$

onde $g$ é a constante de acoplamento dessa teoria. A derivada covariante se transforma como

$$
D_{\mu} \Psi \rightarrow U\left(D_{\mu} \Psi\right)
$$


Temos também que

$$
T^{a} A_{\mu}^{a} \rightarrow U\left(T^{a} A_{\mu}^{a}+\frac{1}{g} \partial_{\mu}\right) U^{-1}
$$

ou, considerando transformações com $\alpha^{a} \ll 1$,

$$
U \simeq 1-i T^{a} \alpha^{a}
$$

obtemos

$$
A_{\mu}^{\prime a}=A_{\mu}^{a}-\frac{1}{g} \partial_{\mu} \alpha^{a}+C_{a b c} \alpha^{b} A_{\mu}^{c},
$$

onde o último termo é resultado da simetria não-abeliana. Nesse caso o tensor $F_{\mu \nu}$ é dado por

$$
F_{\mu \nu}^{a} \equiv \partial_{\mu} A_{\nu}^{a}-\partial_{\nu} A_{\mu}^{a}+g C_{a b c} A_{\mu}^{b} A_{\nu}^{c}
$$

e se transforma como, $F_{\mu \nu}^{\prime a} \rightarrow F_{\mu \nu}^{a}+C_{a b c} \alpha^{b} F_{\mu \nu}^{c}$. Com essas transformações a Lagrangiana de Yang-Mills pode ser escrita como

$$
\mathcal{L}_{\mathrm{A}}=-\frac{1}{4} F_{\mu \nu}^{a} F^{a \mu \nu},
$$

já que o termo de massa $\left(A_{\mu}^{a} A^{a \mu}\right)$ continua não-invariante.

Podemos observar que ao contrário da Lagrangiana da QED (2.10), a Lagrangiana (2.21) possui termos de interação triplos e quárticos. Sendo $F \alpha(\partial A-$ $\partial A)+g A A$, então

$$
\mathcal{L}_{\mathrm{A}} \alpha(\partial A-\partial A)^{2}+g(\partial A-\partial A) A A+g^{2} A A A A
$$




\subsection{Quebra espontânea de simetria}

Para falar do mecanismo de quebra espontânea de simetria podemos ilustrar com o exemplo do ferromagneto com simetria global $S O(3)^{1}$. A Lagrangiana que descreve a interação spin-spin desse sistema é invariante sob rotações tridimensionais, mas seu estado fundamental não.

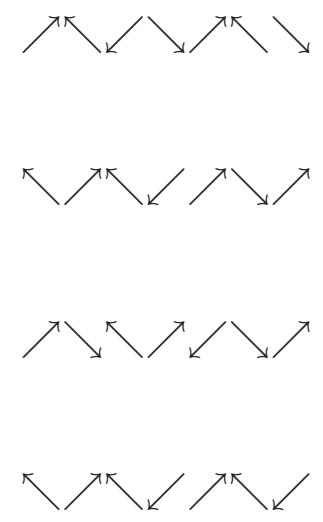

Figura 1.1: Fase paramagnética do ferromagneto com simetria global $\mathrm{SO}(3)$, com temperatura $\mathrm{T}>\mathrm{Tc}$.

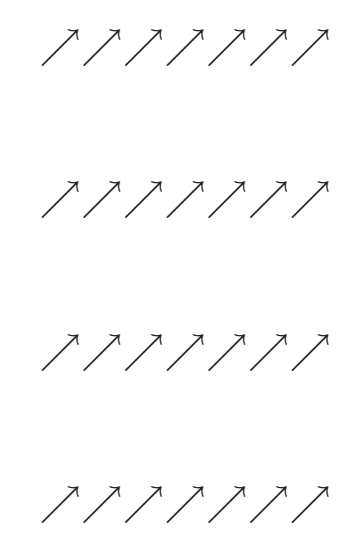

Figura 1.2: Fase ferromagnética com a quebra da simetria global $\mathrm{SO}(3)$ em $\mathrm{SO}(2)$, com temperatura $\mathrm{T}<\mathrm{Tc}$.

Vamos tomar dois exemplos, começando pela teoria $\phi^{4}$, sendo $\phi$ um campo escalar real. A Lagrangiana que descreve essa teoria é dada por

$$
\mathcal{L}_{0}=\frac{1}{2}\left(\partial_{\mu} \phi\right)^{2}+\frac{1}{2} \mu^{2} \phi^{2}-\frac{\lambda}{4 !} \phi^{4} .
$$

onde $m^{2}=-\mu^{2}$, sendo $m$ a massa do campo $\phi$, e $\lambda>0$ uma constante de acoplamento. Essa Lagrangiana é invariante por paridade $(\phi \rightarrow-\phi)$, com o potencial

$$
V(\phi)=-\frac{1}{2} \mu^{2} \phi^{2}+\frac{\lambda}{4 !} \phi^{4}
$$

\footnotetext{
${ }^{1} S O(N)$ é o grupo das matrizes ortogonais $N \times N$ com determinante igual a 1. $S O(3)$ tem simetria de rotação tridimensional, enquanto $S O(2)$ tem simetria de rotação em torno de um único eixo canônico.
} 
Minimizando o potencial, obtemos

$$
\phi_{0}= \pm \sqrt{\frac{6}{\lambda}} \mu= \pm v
$$

onde $v$ é chamado "valor esperado do vácuo" (VEV). Vamos definir o campo $\sigma$, para que o estado de vácuo esteja no zero:

$$
\begin{gathered}
\sigma(x)=\phi(x)-v \rightarrow \phi(x)=\sigma(x)+v, \\
<\sigma>_{0}=0 .
\end{gathered}
$$

Reescrevendo a Lagrangiana $\mathcal{L}_{0} \operatorname{com}(2.26)$,

$$
\mathcal{L}_{0}=\frac{1}{2}\left(\partial_{\mu} \sigma\right)^{2}-\frac{1}{2}\left(2 \mu^{2}\right) \sigma^{2}-\sqrt{\frac{\lambda}{6}} \mu \sigma^{3}-\frac{\lambda}{4 !} \sigma^{4},
$$

que resulta numa Lagrangiana escalar com uma partícula de massa $m_{\sigma}=$ $\sqrt{2} \mu$, e termos de interação cúbicos e quárticos. Dizemos então que a simetria foi quebrada espontaneamente, a simetria está escondida, e é encontrada quando redefinimos o campo físico da Lagrangiana.

Nosso segundo exemplo é uma teoria com simetria global contínua, descrita pela Lagrangiana

$$
\mathcal{L}=\partial_{\mu} \phi^{*} \partial^{\mu} \phi-V\left(\phi^{*} \phi\right)
$$

onde

$$
V\left(\phi^{*} \phi\right)=\mu^{2}\left(\phi^{*} \phi\right)+\lambda\left(\phi^{*} \phi\right)^{2},
$$

sendo que $\phi$ é complexo e $\mathcal{L}$ é invariante sob transformações globais do grupo $U(1)$,

$$
\phi(x) \rightarrow e^{-i \alpha} \phi(x) .
$$


$\operatorname{Com} \rho=\phi^{*} \phi$, temos que

$$
V(\rho)=\mu^{2} \rho+\lambda \rho^{2} .
$$

Redefinindo o campo complexo em termos de campos reais,

$$
\phi=\frac{\left(\phi_{1}+i \phi_{2}\right)}{\sqrt{2}} .
$$

Podemos reescrever a Lagrangiana em termos desses campos como

$$
\mathcal{L}=\frac{1}{2}\left(\partial_{\mu} \phi_{1} \partial^{\mu} \phi_{1}+\partial_{\mu} \phi_{2} \partial^{\mu} \phi_{2}\right)-V\left(\phi_{1}, \phi_{2}\right),
$$

que é invariante sob rotações do grupo $S O(2)$,

$$
\left(\begin{array}{l}
\phi_{1} \\
\phi_{2}
\end{array}\right) \rightarrow\left(\begin{array}{cc}
\cos \theta & -\sin \theta \\
\sin \theta & \cos \theta
\end{array}\right)\left(\begin{array}{l}
\phi_{1} \\
\phi_{2}
\end{array}\right) .
$$

Temos duas possibilidades para esse modelo:

1) $\mu^{2}>0$, com mínimo de potencial em $\phi_{1}=\phi_{2}=0$, temos que,

$$
\mathcal{L}=\sum_{i=1}^{2} \frac{1}{2}\left(\partial_{\mu} \phi_{i} \partial^{\mu} \phi_{i}-\mu^{2} \phi_{i}^{2}\right)
$$

2) $\mu^{2}<0$, minimizando o potencial $\left(\frac{\partial V}{\partial \rho}=0\right)$, encontramos

$$
\rho=|\phi|^{2}=\frac{\phi_{1}^{2}+\phi_{2}^{2}}{2}=-\frac{\mu^{2}}{2 \lambda}=\frac{v^{2}}{2} .
$$

A simetria é quebrada espontaneamente quando escolhemos um vácuo particular, por exemplo

$$
\begin{aligned}
& \phi_{1}=v, \\
& \phi_{2}=0 .
\end{aligned}
$$


Redefinindo os campos,

$$
\begin{gathered}
\phi_{1}^{\prime}=\phi_{1}-v, \\
\phi_{2}^{\prime}=\phi_{2},
\end{gathered}
$$

e substituindo na Lagrangiana (2.33), obtemos

$$
\mathcal{L}=\frac{1}{2} \partial_{\mu} \phi_{1}^{\prime} \partial^{\mu} \phi_{1}^{\prime}+\frac{1}{2} \partial_{\mu} \phi_{2}^{\prime} \partial^{\mu} \phi_{2}^{\prime}-\frac{1}{2}\left(-2 \mu^{2}\right) \phi_{1}^{\prime 2}-\frac{1}{2} 0 \phi_{2}^{\prime 2}+i n t
$$

com dois novos campos escalares $\phi_{1}^{\prime}$ e $\phi_{2}^{\prime}$,

- $\phi_{1}^{\prime}$ com massa $m_{\phi_{1}^{\prime}}^{2}=-2 \mu^{2}$;

- $\phi_{2}^{\prime}$ com massa $m_{\phi_{2}^{\prime}}^{2}=0$ (bóson sem massa).

A aparicão de um estado com $m=0$ é uma consequência do teorema de Goldstone, que estabelece que:

"Se uma simetria global contínua é espontaneamente quebrada, para cada gerador do grupo quebrado, deve aparecer na teoria uma partícula sem massa (bóson de Goldstone)"

\subsection{O Mecanismo de Higgs}

Na última seção nós estudamos o mecanismo de quebra espontânea de simetria em teorias com simetria global. A idéia do mecanismo de Higgs é incluir a quebra espontânea de simetria e a invariância de gauge local numa mesma teoria. Para ilustrar esse mecanismo vamos utilizar dois exemplos, um abeliano e outro não-abeliano. 


\section{Caso Abeliano (Modelo de Ginzburg-Landau)}

A Lagrangiana desse modelo descreve um campo escalar complexo acoplado ao campo eletromagnético,

$$
\mathcal{L}=-\frac{1}{4}\left(F_{\mu \nu}\right)^{2}+\left|D_{\mu} \phi\right|^{2}-V(\phi),
$$

com $D_{\mu}=\partial_{\mu}+i e A_{\mu}$. Essa Lagrangiana é invariante sob a transformação $U(1)$ local,

$$
\begin{gathered}
\phi(x) \rightarrow e^{i \alpha(x)} \phi(x), \\
A_{\mu}(x) \rightarrow A_{\mu}(x)-\frac{1}{e} \partial_{\mu} \alpha(x) .
\end{gathered}
$$

Escolhendo o potencial na forma

$$
V(\phi)=-\mu^{2} \phi^{*} \phi+\frac{\lambda}{2}\left(\phi^{*} \phi\right)^{2} .
$$

Quando $\mu^{2}>0$, a simetria $U$ (1) é quebrada espontaneamente e o campo $\phi$ adquire um VEV dado por

$$
<\phi>=\phi_{0}=\sqrt{\frac{\mu^{2}}{\lambda}} .
$$

Expandindo o campo $\phi(x)$,

$$
\phi(x)=\phi_{0}+\frac{1}{\sqrt{2}}\left(\phi_{1}(x)+i \phi_{2}(x)\right),
$$

e substituindo (2.42) no potencial, usando $\phi_{0}$ dado em (2.41), obtemos

$$
V(\phi)=-\frac{1}{2 \lambda} \mu^{4}+\frac{1}{2}\left(2 \mu^{2}\right) \phi_{1}^{2}+\mathcal{O}\left(\phi^{3}\right) .
$$

Com esse resultado temos que o campo $\phi_{1}$ adquire massa, $m_{\phi_{1}}=\sqrt{2} \mu$, e o campo $\phi_{2}$, permanecendo sem massa, é o bóson de Goldstone do modelo. Agora, substituindo (2.42) no termo cinético da Lagrangiana, 


$$
\left|D_{\mu} \phi\right|^{2}=\frac{1}{2}\left(\partial_{\mu} \phi_{1}\right)^{2}+\frac{1}{2}\left(\partial_{\mu} \phi_{2}\right)^{2}+\sqrt{2} e \phi_{0} A_{\mu} \partial^{\mu} \phi_{2}+e^{2} \phi_{0}^{2} A_{\mu} A^{\mu}+\ldots
$$

onde encontramos um termo de massa para o fóton (campo $A_{\mu}$ nesse modelo),

$$
\triangle \mathcal{L}=\frac{1}{2} m_{A}^{2} A_{\mu} A^{\mu} \rightarrow m_{A}^{2}=2 e^{2} \phi_{0}^{2} .
$$

Podemos notar também que o terceiro termo da equação (2.44) é incoveniente, pois mistura o bóson de gauge com o bóson de goldstone. Esse termo pode ser eliminado fazendo uma escolha de gauge adequada, o chamado gauge unitário,

$$
\alpha(x)=-\frac{\phi_{2}}{e \sqrt{2} \phi_{0}}
$$

Finalmente, a Lagrangiana (2.38) pode ser reescrita como

$$
\mathcal{L}=-\frac{1}{4}\left(F_{\mu \nu}\right)^{2}+\left(\partial_{\mu} \phi\right)^{2}+e^{2} \phi^{2} A_{\mu} A^{\mu}-V(\phi)
$$

Esse modelo explica o efeito Meisner ${ }^{2}$, onde o fóton adquire massa no interior de um supercondutor.

\section{Caso não-Abeliano $(S U(2)$ de gauge acoplado a um campo escalar $\phi$ )}

Nesse caso, temos que a derivada covariante é dada por

$$
D_{\mu} \phi=\left(\partial_{\mu}-i g A_{\mu}^{a} \tau^{a}\right) \phi
$$

onde $\tau^{a}=\frac{\sigma^{a}}{2}$, e $\sigma^{a}$ são as matrizes de Pauli. Usando a liberdade de rotações do grupo $S U(2)$, podemos escrever o valor esperado de $\phi$ na forma

\footnotetext{
${ }^{2}$ Nome que se dá ao fenômeno quando o campo magnético no interior de um supercondutor é zero.
} 


$$
<\phi>=\frac{1}{\sqrt{2}}\left(\begin{array}{l}
0 \\
v
\end{array}\right) .
$$

O termo de massa dos bósons de gauge vem do termo cinético da Lagrangiana,

$$
\left|D_{\mu} \phi\right|^{2}=\frac{1}{2} g^{2}\left(\begin{array}{ll}
0 & v
\end{array}\right) \tau^{a} \tau^{b}\left(\begin{array}{l}
0 \\
v
\end{array}\right) A_{\mu}^{a} A^{b \mu}+\ldots
$$

Simetrizando o produto $\tau^{a} \tau^{b}$, usando $\left\{\tau^{a}, \tau^{b}\right\}=\frac{\delta^{a b}}{2}$, encontramos

$$
\triangle \mathcal{L}=\frac{g^{2} v^{2}}{8} A_{\mu}^{a} A^{a \mu}
$$

Os três bósons de gauge recebem a massa,

$$
m_{A}^{2}=\frac{g^{2} v^{2}}{4}
$$

Uma outra alternativa seria o campo $\phi$ se transformar de acordo com a representação vetorial de $S U(2)$. Nesse caso teríamos

$$
\left(D_{\mu} \phi\right)_{a}=\partial_{\mu} \phi_{a}+g \epsilon_{a b c} A_{\mu}^{b} \phi_{c}
$$

O termo de massa vem de

$$
\triangle \mathcal{L}=\frac{1}{2}\left(D_{\mu} \phi\right)^{2}=\frac{g^{2}}{2}\left(\epsilon_{a b c} A_{\mu}^{b}\left(\phi_{0}\right)_{c}\right)^{2}+\ldots
$$

Escolhendo nossas coordenadas em uma direção particular (3),

$$
<\phi_{c}>=\left(\phi_{0}\right)_{c}=V \delta_{c 3},
$$

e substituindo (2.54) no termo de massa da Lagrangiana (2.53),

$$
\triangle \mathcal{L}=\frac{g^{2} V^{2}}{2}\left(\epsilon_{a b 3} A_{\mu}^{b}\right)^{2}=\frac{g^{2} V^{2}}{2}\left[\left(A_{\mu}^{1}\right)^{2}+\left(A_{\mu}^{2}\right)^{2}\right],
$$

sendo que, somente os bósons correspondentes aos geradores 1 e 2 adquirem massa, 


$$
m_{1}=m_{2}=g V
$$

enquanto o bóson correspondente ao gerador 3 permanece sem massa (já que $\left.b=3 \rightarrow \epsilon_{a 33}=0\right)$. O valor esperado de $\phi_{c}$ destrói a simetria de rotação sobre os eixos 1 e 2, mas preserva a simetria sobre o eixo 3. Portanto os bósons de gauge correspondentes aos geradores não quebrados permanecem sem massa, o que torna o grupo $S U(2)$ um forte candidato à teoria das interações eletrofracas (W's massivos + fóton).

\subsection{Modelo das Interações Eletrofracas}

Muitas foram as tentativas de construir um modelo para as interações eletrofracas. Em 1957 Schwinger [9] propôs o grupo $O(3)$, com um tripleto de campos de gauge $\left(V^{+}, V^{-}, V^{0}\right)$, associado aos bósons carregados e ao fóton. No ano seguinte, Bludman incorporou a estrutura $V-A$ à teoria de gauge com simetria $S U(2)$ isospin, com três bósons de gauge massivos, sendo o bóson massivo neutro associado às correntes neutras que não trocam sabor (observadas primeiramente em 1973), proposto indepententemente por Leite Lopes [10]. Finalmente, em 1961, Glashow [1] sugeriu a possibilidade de incluir ambas as interações, fraca e eletromagnética, no grupo de gauge $S U(2) \otimes U(1)$, onde $U(1)$ foi associado a hipercarga leptônica (Y), relacionada com o isospin (T) e a carga elétrica (Q) pela fórmula de Gell-Mann-Nishijima:

$$
Q=T_{3}+\frac{Y}{2}
$$

Nesse modelo temos quatro bósons de gauge, um tripleto $\left(W^{1}, W^{2}, W^{3}\right)$ associado aos geradores de $S U(2)$ e um singleto $(B)$ associado a $U(1)$. Os bósons carregados $\left(W^{+}, W^{-}\right)$são resultado da combinação linear de $W^{1} \mathrm{e} W^{2}$, enquanto o fóton e o $Z^{0}$ resultam da combinação do $W^{3}$ com o $B$. Os termos de massa dos bósons massivos são colocados "à mão", o que quebra explicitamente a invariança de gauge da teoria. Esse problema foi solucionado com a introdução do mecanismo de Higgs por Salam e Weinberg [2], dando massa 
aos bósons de gauge e preservando a invariança de gauge do modelo. Em 1971 't Hooft $[11,12]$ mostrou que a teoria era renormalizável, consolidando o modelo de Glashow-Weinberg-Salam das interações eletrofracas.

\subsubsection{A estrutura $V-A$}

À altas energias $(E \gg m)$ os spinores de Dirac são autoestados da matriz $\gamma_{5}$ e satisfazem,

$$
\begin{aligned}
& \psi_{L}=\frac{1}{2}\left(1-\gamma_{5}\right) \psi=L \psi, \\
& \psi_{R}=\frac{1}{2}\left(1+\gamma_{5}\right) \psi=R \psi,
\end{aligned}
$$

onde $L$ e $R$ são os operadores projeção de helicidade. Sendo assim, podemos notar que o termo de massa tem a forma

$$
\bar{\psi} \psi=\bar{\psi}_{R} \psi_{L}+\bar{\psi}_{L} \psi_{R}
$$

misturando férmions de mão direita e esquerda, enquanto o vetor corrente eletromagnética é dado por

$$
\bar{\psi} \gamma^{\mu} \psi=\bar{\psi}_{R} \gamma^{\mu} \psi_{R}+\bar{\psi}_{L} \gamma^{\mu} \psi_{L}
$$

sem termos de mistura. A estrutura $(V-A)$ aparece no termo,

$$
\bar{\psi}_{L} \gamma^{\mu} \psi_{L}=\bar{\psi} R \gamma^{\mu} L \psi=\bar{\psi} \gamma^{\mu} L^{2} \psi=\bar{\psi} \gamma^{\mu} L \psi=\frac{1}{2} \bar{\psi} \gamma^{\mu}\left(1-\gamma_{5}\right) \psi
$$

mostrando que somente os férmions de mão-esquerda desempenham um papel importante nas interações fracas carregadas.

\subsubsection{Modelo Eletrofraco}

O grupo de gauge do modelo de Glashow-Weinberg-Salam é 


$$
S U(2)_{L} \otimes U(1)_{Y},
$$

com os geradores satisfazendo a álgebra do grupo:

$$
\left[T^{i}, T^{j}\right]=i \epsilon^{i j k} T^{k} \quad\left[T^{i}, Y\right]=0 .
$$

A Lagrangiana total do modelo é dada por

$$
\mathcal{L}=\mathcal{L}_{\text {Higgs }}+\mathcal{L}_{\text {Gauge }}+\mathcal{L}_{\text {Lép. }}+\mathcal{L}_{\text {Yuk. }} .
$$

A Lagrangiana dos bósons de gauge tem dois termos,

$$
\mathcal{L}_{G}=-\frac{1}{4} W_{\mu \nu}^{i} W^{i \mu \nu}-\frac{1}{4} B_{\mu \nu} B^{\mu \nu},
$$

sendo o primeiro termo associado aos geradores de $S U(2)$, e o segundo associado ao gerador de $U(1)$,

$$
W_{\mu \nu}^{i}=\partial_{\mu} W_{\nu}^{i}-\partial_{\nu} W_{\mu}^{i}-g \epsilon^{i j k} W_{\mu}^{j} W_{\nu}^{k}
$$

e

$$
B_{\mu \nu}=\partial_{\mu} B_{\nu}-\partial_{\nu} B_{\mu} .
$$

A Lagrangiana para os léptons do Modelo Padrão é dividida em duas partes, os campos de mão-direita e de mão esquerda,

$$
\mathcal{L}_{L}=\bar{E}_{R}(i \not D) E_{R}+\bar{E}_{L}(i \not D) E_{L},
$$

onde

$$
E_{R}=e_{R} \quad E_{L}=\left(\begin{array}{c}
\nu_{e} \\
e^{-}
\end{array}\right)_{L}
$$


sendo que o neutrino de mão-direita $\nu_{R}$ não é introduzido no MP. As derivadas covariantes para os campos de mão-direita e de mão-esquerda são dadas por

$$
\begin{gathered}
\bar{E}_{L} i \gamma^{\mu} D_{\mu} E_{L}=\bar{E}_{L} i \gamma^{\mu}\left(\partial_{\mu}+i \frac{g^{\prime}}{2} Y B_{\mu}+i \frac{g}{2} \tau^{i} W_{\mu}^{i}\right) E_{L} \\
\bar{e}_{R} i \gamma^{\mu} D_{\mu} e_{R}=\bar{e}_{R} i \gamma^{\mu}\left(\partial_{\mu}+i \frac{g^{\prime}}{2} Y B_{\mu}\right) e_{R} .
\end{gathered}
$$

A Lagrangiana total para os léptons pode ser dividida em duas partes, o termo cinético e o termo de interação com os bósons de gauge,

$$
\mathcal{L}_{L}=\mathcal{L}_{\text {livre }}+\bar{E}_{L} i \gamma^{\mu}\left(i \frac{g}{2} \tau^{i} W_{\mu}^{i}+i \frac{g^{\prime}}{2} Y B_{\mu}\right) E_{L}+\bar{e}_{R} i \gamma^{\mu}\left(i \frac{g^{\prime}}{2} Y B_{\mu}\right) e_{R}
$$

Considerando agora só os termos de interação entre os léptons e os bósons de gauge, sem os campos de mão-direita,

$$
\begin{gathered}
\mathcal{L}_{L-G}=-g \bar{E}_{L} \gamma^{\mu}\left(\frac{\tau^{1}}{2} W_{\mu}^{1}+\frac{\tau^{2}}{2} W_{\mu}^{2}\right) E_{L} \\
-g \bar{E}_{L} \gamma^{\mu} \frac{\tau^{3}}{2} W_{\mu}^{3} E_{L}-\frac{g^{\prime}}{2} Y \bar{E}_{L} \gamma^{\mu} E_{L} B_{\mu}
\end{gathered}
$$

\section{Correntes carregadas}

Manipulando apenas o primeiro termo da equação (2.66),

$$
\mathcal{L}_{L-G}^{( \pm)}=-\frac{g}{2} \frac{\gamma^{\mu}}{\sqrt{2}}\left(\begin{array}{cc}
\bar{\nu}_{L} & \bar{e}_{L}
\end{array}\right)\left(\begin{array}{cc}
0 & W_{\mu}^{+} \\
W_{\mu}^{-} & 0
\end{array}\right)\left(\begin{array}{c}
\nu_{L} \\
e_{L}
\end{array}\right)
$$

onde $W_{\mu}^{ \pm}=\frac{1}{\sqrt{2}}\left(W_{\mu}^{1} \mp W_{\mu}^{2}\right)$. Essa equação nos dá a descrição das correntes carregadas do modelo padrão,

$$
\mathcal{L}_{L-G}^{( \pm)}=-\frac{g}{2 \sqrt{2}}\left[\bar{\nu} \gamma^{\mu}\left(1-\gamma_{5}\right) e W_{\mu}^{+}+\bar{e} \gamma^{\mu}\left(1-\gamma_{5}\right) \nu W_{\mu}^{-}\right]
$$


reproduzindo a estrutura $(V-A)$ descrita em (2.59).

\section{Correntes neutras}

Podemos agora obter o termo que descreve as correntes neutras do modelo eletrofraco, tomando o segundo e terceiro termo da equação (2.66) e o termo do campo de mão-direita do lépton,

$$
\begin{gathered}
\mathcal{L}_{L-G}^{(0)}=-g \bar{E}_{L}\left(\gamma^{\mu} \frac{\tau^{3}}{2}\right) E_{L} W_{\mu}^{3}-\frac{g^{\prime}}{2}\left(\bar{E}_{L} \gamma^{\mu} Y E_{L}+\bar{e}_{R} \gamma^{\mu} Y e_{R}\right) B_{\mu} \\
=-g J_{3}^{\mu} W_{\mu}^{3}-\frac{g^{\prime}}{2} J_{Y}^{\mu} B_{\mu}
\end{gathered}
$$

onde definimos

$$
\begin{gathered}
J_{3}^{\mu}=\frac{1}{2}\left(\bar{\nu}_{L} \gamma^{\mu} \nu_{L}-\bar{e}_{L} \gamma^{\mu} e_{L}\right), \\
J_{Y}^{\mu}=-\left(\bar{\nu}_{L} \gamma^{\mu} \nu_{L}+\bar{e}_{L} \gamma^{\mu} e_{L}+2 \bar{e}_{R} \gamma^{\mu} e_{R}\right),
\end{gathered}
$$

e usamos $Y_{L}=-1, Y_{R}=-2$, para as hipercargas leptônicas. Podemos notar também que as correntes $J_{3, Y}^{\mu}$ satisfazem a relação de Gell-Mann-Nishijima, $J_{e m}=J_{3}+\frac{1}{2} J_{Y}$. Definindo novos campos $A_{\mu}$ e $Z_{\mu}$ em termos de $W_{\mu}^{3}$ e $B_{\mu}$ :

$$
\left(\begin{array}{c}
A_{\mu} \\
Z_{\mu}
\end{array}\right)=\left(\begin{array}{cc}
\cos \theta_{W} & \operatorname{sen} \theta_{W} \\
-\operatorname{sen} \theta_{W} & \cos \theta_{W}
\end{array}\right)\left(\begin{array}{c}
B_{\mu} \\
W_{\mu}^{3}
\end{array}\right)
$$

$\mathrm{Ou}$

$$
\begin{gathered}
W_{\mu}^{3}=\operatorname{sen} \theta_{W} A_{\mu}+\cos \theta_{W} Z_{\mu}, \\
B_{\mu}=\cos \theta_{W} A_{\mu}-\operatorname{sen} \theta_{W} Z_{\mu},
\end{gathered}
$$

onde $\theta_{W}$ é o ângulo de Weinberg (ou ângulo fraco) e, 


$$
\operatorname{sen} \theta_{W}=\frac{g^{\prime}}{\sqrt{g^{2}+g^{\prime 2}}}, \quad \quad \cos \theta_{W}=\frac{g}{\sqrt{g^{2}+g^{\prime 2}}} .
$$

Reescrevendo a equação (2.69) em termos dos novos campos $A_{\mu}$ e $Z_{\mu}$, obtemos a Lagrangiana que descreve as correntes neutras no Modelo Eletrofraco,

$$
\begin{gathered}
\mathcal{L}_{L-G}^{(0)}=-\left(g \operatorname{sen} \theta_{W} J_{3}^{\mu}+\frac{g^{\prime}}{2} \cos \theta_{W} J_{Y}^{\mu}\right) A_{\mu} \\
+\left(-g \cos \theta_{W} J_{3}^{\mu}+\frac{1}{2} g^{\prime} \operatorname{sen} \theta_{W} J_{Y}^{\mu}\right) Z_{\mu} \\
\mathcal{L}_{L-G}^{(0)}=-g \operatorname{sen} \theta_{W}\left(\bar{e} \gamma^{\mu} e\right) A_{\mu}-\frac{g}{2 \cos \theta_{W}} \sum_{\psi_{i}=\nu, l} \bar{\psi}_{i} \gamma^{\mu}\left(g_{V}^{i}-g_{A}^{i} \gamma_{5}\right) \psi_{i} Z_{\mu}
\end{gathered}
$$

onde

$$
e=g \operatorname{sen} \theta_{W}=g^{\prime} \cos \theta_{W}
$$

e

$$
g_{V}^{i} \equiv T_{3}^{i}-2 Q_{i} \operatorname{sen}^{2} \theta_{W} \quad g_{A}^{i} \equiv T_{3}^{i}
$$

As correntes neutras foram confirmadas experimentamente em 1973.

\section{O Mecanismo de Higgs}

As Lagrangianas (2.68) e (2.72) nos descrevem todas as interações fundamentais do ME, restando agora introduzir o mecanismo de Higgs, já que todas as partículas, bósons de gauge e léptons, ainda permanecem sem massa. Para isso precisamos introduzir uma partícula escalar na teoria, o bóson de Higgs, como um dubleto de $S U(2)$, 


$$
\begin{gathered}
\Phi=\left(\begin{array}{c}
\phi^{+} \\
\phi^{0}
\end{array}\right), \\
\mathcal{L}_{H}=\left(D^{\mu} \Phi\right)^{\dagger}\left(D_{\mu} \Phi\right)-V\left(\Phi^{\dagger} \Phi\right),
\end{gathered}
$$

com o potencial na forma

$$
V\left(\Phi^{\dagger} \Phi\right)=\mu^{2} \Phi^{\dagger} \Phi+\lambda\left(\Phi^{\dagger} \Phi\right)^{2}
$$

A simetria é quebrada espontaneamente quando $\mu^{2}<0$, onde o valor esperado do campo do Higgs pode ser escolhido como

$$
<\Phi>_{0}=\frac{v}{\sqrt{2}}\left(\begin{array}{l}
0 \\
1
\end{array}\right)
$$

onde $v=\sqrt{-\frac{\mu^{2}}{\lambda}}$. O grupo de simetria do modelo é quebrado em

$$
S U(2)_{L} \otimes U(1)_{Y} \rightarrow U(1)_{e m}
$$

A condição que deixa o vácuo invariante é

$$
G<\Phi>_{0}=0
$$

Para os quatro geradores de $S U(2)_{L} \otimes U(1)_{Y}$ temos que

$$
\tau_{1}<\Phi>_{0} \neq 0, \quad \tau_{2}<\Phi>_{0} \neq 0, \quad \tau_{3}<\Phi>_{0} \neq 0, \quad Y<\Phi>_{0} \neq 0,
$$

mas

$$
Q<\Phi>_{0}=\frac{1}{2}\left(\tau_{3}+Y\right)=0,
$$

o que faz com que o fóton permaneça sem massa, enquanto os outros bósons de gauge devem adquirir massa, de acordo com o teorema de Goldstone e o 
mecanismo de Higgs. Parametrizando o campo $\Phi$,

$$
\begin{gathered}
\Phi=U(\chi)\left(\begin{array}{c}
0 \\
\frac{(v+h)}{\sqrt{2}}
\end{array}\right)=\exp \left(\frac{i \chi \cdot \tau}{2 v}\right)\left(\begin{array}{c}
0 \\
\frac{(v+h)}{\sqrt{2}}
\end{array}\right) \\
=\frac{1}{\sqrt{2}}\left(\begin{array}{c}
i \sqrt{2} \omega^{+} \\
v+h-i z^{0}
\end{array}\right),
\end{gathered}
$$

onde $\omega^{ \pm}$e $z^{0}$ são os bósons de Goldstone. Agora podemos escolher o gauge unitário $\left(\alpha^{i}=\chi^{i} / v\right)$, com as seguintes transformações:

$$
\begin{gathered}
\Phi \rightarrow \Phi^{\prime}=U^{-1}(\chi) \Phi=\frac{1}{\sqrt{2}}\left(\begin{array}{c}
0 \\
v+h
\end{array}\right), \\
\tau \cdot W_{\mu} \rightarrow \tau \cdot W_{\mu}^{\prime}, \\
B_{\mu} \rightarrow B_{\mu}, \\
E_{R} \rightarrow E_{R}, \\
E_{L} \rightarrow E_{L}^{\prime}=U^{-1}(\chi) E_{L} .
\end{gathered}
$$

Sendo a lagrangeana de Yukawa para os léptons dada por

$$
\mathcal{L}_{Y}=-G_{l}\left[\bar{E}_{R}\left(\Phi^{\dagger} E_{L}\right)+\left(\bar{E}_{L} \Phi\right) E_{R}\right]
$$

Com as transformações de gauge acima podemos reescrevê-la como

$$
\mathcal{L}_{Y}=-G_{l} \frac{v+h}{\sqrt{2}}\left(\bar{e}_{R} e_{L}+\bar{e}_{L} e_{R}\right)=-G_{l} \frac{v+h}{\sqrt{2}} \bar{e} e .
$$

Obtemos então que a massa do lépton (elétron, múon, tau) ${ }^{3}$ é

\footnotetext{
${ }^{3}$ Essas partículas com seus respectivos neutrinos contituem as três gerações de léptons
} 


$$
m_{l}=\frac{G_{l} v}{\sqrt{2}}
$$

A massa dos bósons de gauge pode ser obtida da Lagrangiana (2.74),

$$
\begin{aligned}
\mathcal{L}_{H}=\left|D_{\mu} \Phi\right|^{2}-V\left(|\Phi|^{2}\right) & =\left|\left(\partial_{\mu}+i \frac{g^{\prime}}{2} Y B_{\mu}+i \frac{g}{2} \tau^{i} W_{\mu}^{i}\right) \frac{(v+h)}{\sqrt{2}}\left(\begin{array}{l}
0 \\
1
\end{array}\right)\right|^{2} \\
& -\mu^{2} \frac{(v+h)^{2}}{2}-\lambda \frac{(v+h)^{4}}{4}
\end{aligned}
$$

Com algumas manipulações encontramos

$$
\begin{aligned}
\mathcal{L}_{H}= & \frac{1}{2} \partial_{\mu} h \partial^{\mu} h-\frac{1}{2}\left(2 \mu^{2}\right) h^{2}+\frac{g^{2} v^{2}}{4} W_{\mu}^{+} W^{\mu-} \\
& +\frac{g^{2} v^{2}}{8 \cos ^{2} \theta_{W}} Z_{\mu} Z^{\mu}+\mathcal{O} A_{\mu} A^{\mu}+\ldots,
\end{aligned}
$$

onde

$$
\begin{array}{cc}
W_{\mu}^{ \pm}=\frac{1}{\sqrt{2}}\left(W_{\mu}^{1} \mp i W_{\mu}^{2}\right), & m_{W}=\frac{g v}{2}, \\
Z_{\mu}=\frac{1}{\sqrt{g^{2}+g^{\prime 2}}}\left(g W_{\mu}^{3}-g^{\prime} B_{\mu}\right), & m_{Z}=\sqrt{g^{2}+g^{\prime 2}} \frac{v}{2}, \\
A_{\mu}=\frac{1}{\sqrt{g^{2}+g^{\prime 2}}}\left(g^{\prime} W_{\mu}^{3}+g B_{\mu}\right), & m_{A}=0, \\
m_{H}=\sqrt{-2 \mu^{2}}=\sqrt{2 \lambda} v . &
\end{array}
$$

Para obtermos os valores teóricos das massas dessas patículas precisamos conhecer as constantes de acoplamento e o VEV do Higgs. Já sabemos que

do Modelo Padrão, sendo que os neutrinos não adquirem massa pelo mecanismo de Higgs, e suas massas não são explicadas pela teoria. 


$$
e=\frac{g g^{\prime}}{\sqrt{g^{2}+g^{\prime 2}}}=g \operatorname{sen} \theta_{W}=g^{\prime} \cos \theta_{W}
$$

e usando a relação

$$
\frac{g}{2 \sqrt{2}}=\left(\frac{m_{W}^{2} G_{F}}{\sqrt{2}}\right)^{1 / 2} .
$$

Substituindo $m_{W}$ nessa equação, encontramos

$$
v=\left(\sqrt{2} G_{F}\right)^{-1 / 2} \simeq 246 G e V
$$

Agora podemos encontrar $g$ a partir da equação (2.89) e do valor experimental $\operatorname{sen}^{2} \theta_{W} \simeq 0.23$ [13], obtendo

$$
g=\frac{e}{\operatorname{sen} \theta_{W}}=\frac{\sqrt{4 \pi \alpha}}{\operatorname{sen} \theta_{W}} \simeq 0.65
$$

Com esses valores obtemos

$$
\begin{gathered}
m_{W} \simeq 80 \mathrm{GeV}, \\
m_{Z}=\frac{m_{W}}{\cos \theta_{W}} \simeq 91 \mathrm{GeV},
\end{gathered}
$$

sendo que a massa do Higgs permanece indeterminada, já que desconhecemos o valor da constante $\lambda$. Esses valores estão de acordo com os dados experimentais, assim como a previsão para o parâmetro $\rho$ a nível árvore,

$$
\rho=\frac{m_{W}^{2}}{\cos ^{2} \theta_{W} m_{Z}^{2}}=1,
$$

que demonstram os resultados bem sucedidos do MP. 


\subsection{QCD}

Para completarmos o MP é necessário introduzir os hádrons (bárions e mésons), partículas que interagem através da interação forte, a interação mais intensa do modelo. Os hádrons são formados pelos quarks, que interagem através dos glúons. Assim como os léptons, os quarks são partículas elementares que podem sentir as interações fraca (sabor) e eletromagnética (carga), e sendo assim podemos extender o modelo eletrofraco a essas partículas.

\subsubsection{Introduzindo os Quarks}

Em 1961 foi observado que os decaimentos hadrônicos fracos relacionados a estranheza são muito suprimidos pela natureza. Até aquela época eram conhecidos 3 sabores de quarks, u (up), d (down) e s (strange), que podem ser colocados na forma de um dubleto,

$$
L_{U}=\left(\begin{array}{c}
u \\
d^{\prime}
\end{array}\right)_{L}=\left(\begin{array}{c}
u \\
\cos \theta_{C} d+\operatorname{sen} \theta_{C} s
\end{array}\right)_{L},
$$

onde $\theta_{C}$ é o ângulo de Cabibbo. Dessa forma a corrente hadrônica neutra pode ser escrita como

$$
\begin{gathered}
J_{\mu}^{H}(0)=\bar{u} \gamma_{\mu}\left(1-\gamma_{5}\right) u+\bar{d}^{\prime} \gamma_{\mu}\left(1-\gamma_{5}\right) d^{\prime} \\
=\bar{u} \gamma_{\mu}\left(1-\gamma_{5}\right) u+\cos ^{2} \theta_{C} \bar{d} \gamma_{\mu}\left(1-\gamma_{5}\right) d+\operatorname{sen}^{2} \theta_{C} \bar{s} \gamma_{\mu}\left(1-\gamma_{5}\right) s \\
+\cos \theta_{C} \operatorname{sen} \theta_{C}\left[\bar{d} \gamma_{\mu}\left(1-\gamma_{5}\right) s+\bar{s} \gamma_{\mu}\left(1-\gamma_{5}\right) d\right],
\end{gathered}
$$

sendo o último termo responsável pela mudança de sabor nas correntes neutras (FCNC). Os resultados experimentais obtidos para decaimento de kaons via correntes carregadas são dados por 


$$
B R\left(K_{u \bar{s}}^{+} \rightarrow W^{+} \rightarrow \mu^{+} \nu\right) \simeq 63.5 \%
$$

enquanto processos envolvendo FCNC são muito suprimidos

$$
\begin{aligned}
& B R\left(K_{u \bar{s}}^{+} \rightarrow \pi_{u \bar{d}}^{+} \nu \bar{\nu}\right) \simeq 4.2 \times 10^{-10}, \\
& B R\left(K_{d \bar{s}}^{L} \rightarrow \mu^{+} \mu^{-}\right) \simeq 7.2 \times 10^{-9}
\end{aligned}
$$

A solução para esse problema foi proposta em 1970 por Glashow, Iliopoulos e Maiani (mecanismo GIM) [14], que consideraram um quarto sabor de quark, o quark c (charm), completando a simetria entre quarks e léptons conhecidos na época,

$$
\begin{gathered}
L_{U}=\left(\begin{array}{c}
u \\
d^{\prime}
\end{array}\right)_{L}=\left(\begin{array}{c}
u \\
\cos \theta_{C} d+\operatorname{sen} \theta_{C} s
\end{array}\right)_{L}, \\
L_{C}=\left(\begin{array}{c}
c \\
s^{\prime}
\end{array}\right)_{L}=\left(\begin{array}{c}
c \\
-\operatorname{sen} \theta_{C} d+\cos \theta_{C} s
\end{array}\right)_{L},
\end{gathered}
$$

e os respectivos singletos de mão direita,

$$
R_{U} \quad, \quad R_{D} \quad, \quad R_{C}, R_{S}
$$

Os quarks podem ser introduzidos no Modelo Padrão de forma semelhante aos léptons. A lagrangeana para os quarks sem massa é dada por

$$
\mathcal{L}_{q}=\bar{L}_{U} i \not D L_{U}+\bar{L}_{C} i \not D L_{C}+\bar{R}_{U} i \not D R_{U}+\ldots+\bar{R}_{S} i \not D R_{S}
$$

onde os acoplamentos com os bósons de gauge vem da derivada covariante,

$$
L: D_{\mu} \rightarrow \partial_{\mu}+i \frac{g^{\prime}}{2} Y B_{\mu}+i \frac{g}{2} \tau^{i} W_{\mu}^{i}
$$




$$
R: D_{\mu} \rightarrow \partial_{\mu}+i \frac{g^{\prime}}{2} Y B_{\mu}
$$

As hipercargas leptônicas podem ser obtidas pela fórmula de Gell-MannNishijima $\left(Y=2\left(Q-T_{3}\right)\right)$, conhecidas a carga $Q\left(Q_{U, C}=+\frac{2}{3}, Q_{D, S}=-\frac{1}{3}\right)$ e a terceira componente de isospin $T_{3}\left(T_{3}^{L}= \pm \frac{1}{2}, T_{3}^{R}=0\right)$, encontramos

$$
Y_{L_{Q}}=\frac{1}{3} \quad, \quad Y_{R_{U}, R_{C}}=\frac{4}{3} \quad, \quad Y_{R_{D}, R_{S}}=-\frac{2}{3}
$$

Usando esses resultados na lagrangeana dos quarks podemos obter as correntes carregadas e neutras,

$$
\mathcal{L}_{q}^{( \pm)}=\frac{g}{2 \sqrt{2}}\left[\bar{u} \gamma^{\mu}\left(1-\gamma_{5}\right) d^{\prime}+\bar{c} \gamma^{\mu}\left(1-\gamma_{5}\right) s^{\prime}\right] W_{\mu}^{+}+\text {h.c. }
$$

As correntes neutras ficam proporcionais a

$$
\bar{c} \gamma^{\mu}\left(1-\gamma_{5}\right) c+\bar{s}^{\prime} \gamma^{\mu}\left(1-\gamma_{5}\right) s^{\prime}
$$

evitando o problema da FCNC. A interação dos quarks com o bóson de gauge neutro $Z$ é dada por

$$
\mathcal{L}_{q}^{(0)}=-\frac{g}{2 \cos \theta_{W}} \sum_{\psi_{q}=u, \ldots, s} \bar{\psi}_{q} \gamma^{\mu}\left(g_{V}^{q}-g_{A}^{q} \gamma_{5}\right) \psi_{q} Z_{\mu}
$$

\subsubsection{Anomalias}

Na teoria quântica de campos algumas correções radiativas em loop podem violar a conservação da simetria local, derivada da invariância de gauge. Essas correções em loop que quebram as identidades de Ward-Takahashi, invalidando a prova de renormalizabilidade, são chamadas de anomalias, e aparecem no modelo GWS (Glashow-Weinberg-Salam) na forma de diagramas triângulo (Fig. 2.2). A teoria vai ser livre de anomalias se

$$
\operatorname{tr}\left[\gamma^{5} t^{a}\left\{t^{b}, t^{c}\right\}\right]=0
$$


Essa condição só é satisfeita para todos os diagramas da figura (2.2) quando organizamos os quarks e léptons em multipletos e adicionamos um novo número quântico para os quarks, a carga de "cor", associada ao grupo $S U(3)$. Sendo assim, com a descoberta do lépton $\tau$ (1975) e do quark $b$ (1977) os férmions foram organizados em multipletos, sendo os quarks ainda tripletos de cor (azul, verde, vermelho),

$$
\begin{aligned}
& \left\{\left(\begin{array}{c}
\nu_{e} \\
e
\end{array}\right)_{L}, e_{R},\left(\begin{array}{l}
u \\
d
\end{array}\right)_{L}, u_{R}, d_{R}\right\} \\
& \left\{\left(\begin{array}{c}
\nu_{\mu} \\
\mu
\end{array}\right)_{L}, \mu_{R},\left(\begin{array}{c}
c \\
s
\end{array}\right)_{L}, c_{R}, s_{R}\right\} \\
& \left\{\left(\begin{array}{c}
\nu_{\tau} \\
\tau
\end{array}\right)_{L}, \tau_{R},\left(\begin{array}{c}
t \\
b
\end{array}\right)_{L}, t_{R}, b_{R}\right\}
\end{aligned}
$$

Este foi um forte argumento em favor da existência do quark top, o último quark descoberto somente em 1995.
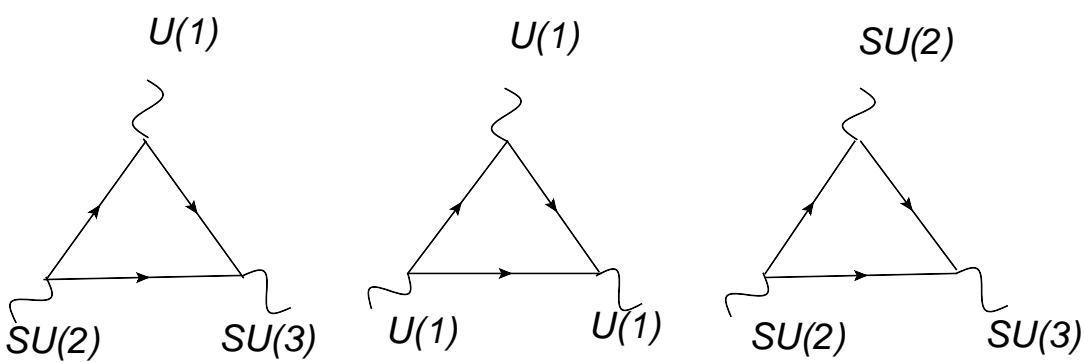

Figura 2.2. Possíveis anomalias de gauge na teoria das interações eletrofracas.

\subsubsection{Massa dos Quarks e Matriz CKM}

Para gerar a massa dos quarks precisamos de um dubleto de Higgs com $Y=-1$. Partindo da equação (2.73), definimos um dubleto conjugado com a seguinte forma 


$$
\tilde{\Phi}=i \sigma_{2} \Phi^{*}=\left(\begin{array}{c}
\phi^{0 *} \\
-\phi^{-}
\end{array}\right)
$$

De forma análoga aos léptons podemos escrever a Lagrangiana para os acoplamentos de Yukawa considerando as três gerações de quark,

$$
\mathcal{L}_{Y}^{q}=-\sum_{i, j=1}^{3}\left[G_{i j}^{U} \bar{R}_{U_{i}}\left(\tilde{\Phi}^{\dagger} L_{j}\right)+G_{i j}^{D} \bar{R}_{D_{i}}\left(\Phi^{\dagger} L_{j}\right)+\text { h.c. }\right],
$$

onde $U_{i}=u, c, t$ e $D_{i}=d, s, b$.

Os termos de massa podem ser obtidos usando os valores esperados de $\Phi$ e $\tilde{\Phi}$

$$
\begin{aligned}
& \left(\bar{u}^{\prime} \bar{c}^{\prime} \bar{t}^{\prime}\right)_{R} \mathcal{M}^{U}\left(\begin{array}{l}
u^{\prime} \\
c^{\prime} \\
t^{\prime}
\end{array}\right)_{L}+\text { h.c. } \\
& \left(\bar{d}^{\prime} \bar{s}^{\prime} \bar{b}^{\prime}\right)_{R} \mathcal{M}^{D}\left(\begin{array}{c}
d^{\prime} \\
s^{\prime} \\
b^{\prime}
\end{array}\right)_{L}+\text { h.c. }
\end{aligned}
$$

onde $\mathcal{M}_{i j}^{U(D)}=\left(\frac{v}{\sqrt{2}}\right) G_{i j}^{U(D)}$ são matrizes de massa não-diagonais.

Os autoestados físicos $\left(q^{\prime}\right)$ são superposições lineares dos autoestados de massa $(q)$ dados pelas transformações unitárias:

$$
\left(\begin{array}{c}
u^{\prime} \\
c^{\prime} \\
t^{\prime}
\end{array}\right)=U_{L, R}\left(\begin{array}{c}
u \\
c \\
t
\end{array}\right) \quad, \quad\left(\begin{array}{c}
d^{\prime} \\
s^{\prime} \\
b^{\prime}
\end{array}\right)=D_{L, R}\left(\begin{array}{c}
d \\
s \\
b
\end{array}\right),
$$

sendo $U_{L, R}$ e $D_{L, R}$ matrizes untárias que diagonalizam as matrizes de massa,

$$
U_{R}^{-1} \mathcal{M}^{U} U_{L}=\left(\begin{array}{ccc}
m_{u} & 0 & 0 \\
0 & m_{c} & 0 \\
0 & 0 & m_{d}
\end{array}\right)
$$




$$
D_{R}^{-1} \mathcal{M}^{D} D_{L}=\left(\begin{array}{ccc}
m_{d} & 0 & 0 \\
0 & m_{s} & 0 \\
0 & 0 & m_{b}
\end{array}\right) .
$$

Agora podemos escrever as correntes carregadas, que são proporcionais a

$$
\left(\bar{u}^{\prime} \bar{c}^{\prime} \bar{t}^{\prime}\right)_{L} \gamma^{\mu}\left(\begin{array}{c}
d^{\prime} \\
s^{\prime} \\
b^{\prime}
\end{array}\right)_{L}=(\bar{u} \bar{c} \bar{t})_{L}\left(U_{L}^{\dagger} D_{L}\right) \gamma^{\mu}\left(\begin{array}{c}
d \\
s \\
b
\end{array}\right)_{L},
$$

onde $V=U_{L}^{\dagger} D_{L}$ é a matriz CKM (Cabibbo-Kobayashi-Maskawa), que mistura os autoestados de massa das diferentes gerações. Por outro lado, as correntes neutras são proporcionais a

$$
\begin{aligned}
& \left(\bar{u}^{\prime} \bar{c}^{\prime} \bar{t}^{\prime}\right)_{L} \gamma^{\mu}\left(\begin{array}{c}
u^{\prime} \\
c^{\prime} \\
t^{\prime}
\end{array}\right)_{L}=(\bar{u} \bar{c} \bar{t})_{L}\left(U_{L}^{\dagger} U_{L}\right) \gamma^{\mu}\left(\begin{array}{c}
u \\
c \\
t
\end{array}\right)_{L} \\
& \left(\bar{d}^{\prime} \bar{s}^{\prime} \bar{b}^{\prime}\right)_{L} \gamma^{\mu}\left(\begin{array}{c}
d^{\prime} \\
s^{\prime} \\
b^{\prime}
\end{array}\right)_{L}=(\bar{d} \bar{s} \bar{b})_{L}\left(D_{L}^{\dagger} D_{L}\right) \gamma^{\mu}\left(\begin{array}{c}
d \\
s \\
b
\end{array}\right)_{L},
\end{aligned}
$$

não ocorrendo mistura de sabores nesse setor (FCNC), já que $U_{L}^{\dagger} U_{L}=$ $D_{L}^{\dagger} D_{L}=1$.

\begin{tabular}{|c|c|}
\hline Partícula & Massa \\
\hline \hline$W^{ \pm}$ & $80,398 \pm 0,025 \mathrm{GeV}$ \\
\hline$Z^{0}$ & $91,1876 \pm 0,0021 \mathrm{GeV}$ \\
\hline$\gamma$ [fóton] & $<1 \times 10^{-18} \mathrm{eV}$ \\
\hline$g$ [glúon] & 0 \\
\hline$h$ [Higgs] & $>114,4 \mathrm{GeV}$ \\
\hline
\end{tabular}

Figura 2.1. Massa dos bósons de gauge do MP (Particle Data Group, PDG) [13]. 


\subsection{Modelo Padrão e suas interações}

Como vimos nas últimas seções a Lagrangiana do Modelo Padrão é composta de três tipos de campos fundamentais; o campo fermiônico (quarks e léptons), o campo de gauge (bósons eletrofracos e o fóton) e um campo escalar (o bóson de Higgs), responsável pelo mecanismo de quebra espontânea de simetria que dá massa a todas as partículas do modelo. Vimos também que os férmions podem interagir entre si, e essas interações são mediadas pelos bósons do modelo (fóton, $Z, W^{ \pm}$, e $h$ ). A Lagrangiana total do modelo pode ser escrita como

$$
\mathcal{L}=\mathcal{L}_{\text {Higgs }}+\mathcal{L}_{\text {Gauge }}+\mathcal{L}_{\text {Férm. }}+\mathcal{L}_{\text {Yuk. }} .
$$

Para descrevermos todas as interações do Modelo Padrão vamos dividir a Lagrangiana total em três partes: gauge + Higgs, quarks + Yukawa e léptons + Yukawa.

\section{Bósons de Gauge + Higgs:}

$$
\begin{gathered}
\mathcal{L}_{G}+\mathcal{L}_{H}=-\frac{1}{4} F_{\mu \nu} F^{\mu \nu}-\frac{1}{2} W_{\mu \nu}^{+} W^{-\mu \nu}+m_{W}^{2} W_{\mu}^{+} W^{\mu-}-\frac{1}{4} Z_{\mu \nu} Z^{\mu \nu}+m_{Z}^{2} Z_{\mu} Z^{\mu} \\
+\frac{1}{2} \partial_{\mu} H \partial^{\mu} H-\frac{1}{2} m_{H}^{2} H^{2}+W^{+} W^{-} A+W^{+} W^{-} Z+W^{+} W^{-} A A+ \\
+W^{+} W^{-} Z Z+W^{+} W^{-} A Z+W^{+} W^{-} W^{+} W^{-}+H H H+H H H H \\
+W^{+} W^{-} H+W^{+} W^{-} H H+Z Z H+Z Z H H .
\end{gathered}
$$

\section{Léptons + Yukawa:}

$\mathcal{L}_{L}+\mathcal{L}_{Y}=\bar{e}_{R} i \gamma^{\mu}\left(\partial_{\mu}+i \frac{g^{\prime}}{2} Y B_{\mu}\right) e_{R}+\bar{E}_{L} i \gamma^{\mu}\left(\partial_{\mu}+i \frac{g^{\prime}}{2} Y B_{\mu}+i \frac{g}{2} \tau^{i} W_{\mu}^{i}\right) E_{L}$ 


$$
\begin{gathered}
-G_{l}\left[\bar{e}_{R}\left(\Phi^{\dagger} E_{L}\right)+\left(\bar{E}_{L} \Phi\right) e_{R}\right]=\sum_{l=e, \mu, \tau} \bar{l}\left(i \not \partial-m_{l}\right) l+\sum_{\nu_{l}=\nu_{e}, \nu_{\mu}, \nu_{\tau}} \bar{\nu}_{l}(i \not \partial) \nu_{l}+\operatorname{massa}\left(\nu_{l}\right) \\
+\bar{l} l A+\bar{\nu}_{l} l W^{+} \bar{l}_{l} W^{-}+\bar{l} l Z+\bar{\nu}_{l} \nu_{l} Z+\bar{l} l H .
\end{gathered}
$$

\section{Quarks + Yukawa:}

$$
\begin{gathered}
\mathcal{L}_{q}+\mathcal{L}_{Y}=\sum_{i, j=1}^{3}\left[\bar{L}_{j} i \not D L_{j}+\bar{R}_{U_{i}} i \not D R_{U_{i}}+\bar{R}_{D_{i}} i \not D R_{D_{i}}\right. \\
\left.-\left(G_{i j}^{U} \bar{R}_{U_{i}}\left(\tilde{\Phi}^{\dagger} L_{j}\right)+G_{i j}^{D} \bar{R}_{D_{i}}\left(\Phi^{\dagger} L_{j}\right)+\text { h.c. }\right)\right] \\
=\sum_{q=u, \ldots, t} \bar{q}\left(i \not \partial-m_{q}\right) q+\bar{q} q A+\bar{u} d^{\prime} W^{+}+\bar{d}^{\prime} u W^{-}+\bar{q} q Z+\bar{q} q H .
\end{gathered}
$$

A observação da maior parte dessas interações mostra o grande sucesso de predição do Modelo Padrão, no entanto permanece um grande desafio para o modelo a busca pelo bóson de Higgs, a partícula responsável pela quebra de simetria na teoria. Esse é o principal objetivo do LHC (Large Hadron Colider), que começou a funcionar em 2008. No próximo capítulo apresentaremos os principais problemas do MP, e também discutiremos a possibilidade de nova física além do MP na escala $\mathrm{TeV}$ (escala de energia a ser explorada pelo LHC), relacionada com a origem da quebra da simetria eletrofraca. 


\section{Capítulo 3}

\section{Além do Modelo Padrão}

Nos últimos quarenta anos o MP se mostrou bem sucedido, em concordância com os dados experimentais. Desde a observação das correntes neutras na década de 70, muitas outras descobertas contribuíram para o fortalecimento do modelo, principalmente a observação dos bósons responsáveis pela interação fraca e suas massas, em pleno acordo com a teoria. No entanto, a partícula responsável por gerar as massas de todas as outras partículas do modelo, o bóson de Higgs, ainda não foi encontrada. Esse é um dos objetivos do LHC, o grande acelerador de hádrons, que tem como objetivo não só a busca pela partícula responsável pela quebra de simetria do MP, como também a procura de nova física além do MP. O fato é que esse modelo permanece incompleto e contém alguns problemas importantes, como o problema da hierarquia, a origem da quebra da simetria eletrofraca (EWSB, Electroweak Symmetry Breaking), o problema da hierarquia da massa dos férmions, o problema da constante cosmológica, quais as partículas que formam a matéria escura, o problema da violação de CP nas interações fortes, a assimetria matériaantimatéria, etc. Entre as teorias que propõem soluções para alguns desses problemas podemos citar, supersimetria, teorias fortemente acopladas (technicolor, topcolor) e as teorias com dimensões extras (LED, UED e WED). O presente trabalho tem como objetivo estudar a fenomenologia no LHC de modelos de quebra da simetria eletrofraca por condensação de uma quarta geração, modelos que buscam resolver dois desses problemas fundamentais do 
MP (problema da hierarquia de escalas relacionado com a origem da EWSB, e a hierarquia das massas fermiônicas) utilizando uma combinação de duas dessas teorias além do MP, partindo da correpondência AdS/CFT, teorias fortemente acopladas e teorias com dimensões extras curvas (WED, Warped Extra Dimensions). Nesse capítulo mostraremos os principais problemas do Modelo Padrão e os requisitos básicos para a construção de um modelo de quebra da simetria eletrofraca.

\subsection{Problemas do MP}

\subsubsection{Problema da Hierarquia}

No Modelo Padrão a massa das partículas é a soma da sua massa nua com a correção radiativa $\left(m=m_{0}+\delta m\right)$. Na QED a massa do elétron a um loop é dada por

$$
m_{e}=m_{0 e}+\frac{3 m_{0 e} \alpha}{2 \pi} \ln \left(\frac{\Lambda}{m_{0 e}}\right)
$$

Sendo assim, o elétron (ou qualquer férmion carregado) recebe correções a um loop que são logariticamente divergentes e proporcionais a sua massa nua. Portanto, no limite $m_{0_{e}} \rightarrow 0$, a massa corrigida permanece zero (simetria quiral), e para $m_{0_{e}} \neq 0$ a divergência é só logaritimica, então mesmo para grandes valores de $\Lambda$, a correção para a massa da partícula a um loop será relativamente pequena, da ordem $\sim 1$. Assim como a massa dos férmions está protegida por uma simetria, a simetria quiral, a massa dos bósons também está protegida por uma simetria, a simetria de gauge. No entanto, não existe nenhuma simetria que proteja o bóson de Higgs, a partícula escalar da teoria. Exemplos de correções radiativas para a massa do Higgs são dadas pelos diagramas da figura (3.1) [15]. Calculando a correção para o primeiro diagrama, encontramos

$$
-i \Sigma_{H}=-i \lambda \int_{0}^{\Lambda} \frac{d^{4} k}{(2 \pi)^{4}} \frac{i}{k^{2}-m^{2}} \approx \frac{\lambda}{16 \pi^{2}} \Lambda^{2}
$$


O cálculo da contribuição do segundo diagrama, com um loop de férmions (contribuição do top), fornece

$$
\begin{gathered}
=(-1)(-i \lambda)^{2} N_{c} \int_{0}^{\Lambda} \frac{d^{4} k}{(2 \pi)^{4}} \operatorname{Tr}\left[\frac{\left(1-\gamma^{5}\right)}{2} \frac{i(\not k+m)}{\left(k^{2}-m^{2}\right)} \frac{\left(1+\gamma^{5}\right)}{2} \frac{i(\not p+\not k+m)}{\left((p+k)^{2}-m^{2}\right)}\right] \\
\approx-\frac{3 \lambda^{2}}{8 \pi^{2}} \Lambda^{2}
\end{gathered}
$$

Esses resultados mostram que a correção radiativa à massa do Higgs depende quadraticamente do "cutoff" da teoria, o que significa que o MP não pode ser válido até a escala de Planck $\left(\Lambda \sim 10^{19} \mathrm{GeV}\right)$, já que a massa do Higgs deve ser no máximo da ordem de $1 \mathrm{TeV}$. A correção da massa da partícula deve ser da mesma ordem ou menor, então devemos esperar que a escala da nova física seja a escala $\mathrm{TeV}$. Antes de prosseguirmos podemos mostrar como se obtém esse limite superior para a massa do Higgs, sendo que o limite inferior obtido experimentalmente é de $114 \mathrm{GeV}$ (PDG) [13].

a)

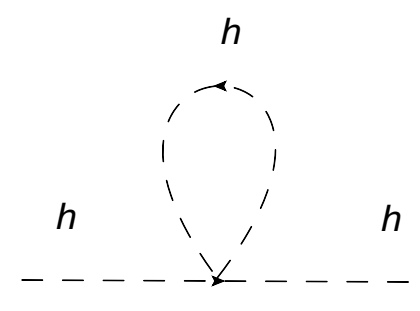

b)

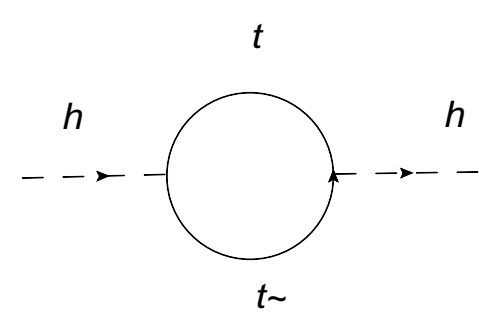

Figura 3.1. Correções radiativas à massa do Higgs: a) auto-energia do Higgs;

b) loop de férmions (quark top).

\section{Massa do Higgs}

Um limite superior para a massa do Higgs pode ser obtido do espalhamento $W_{L}^{+} W_{L}^{-} \rightarrow W_{L}^{-} W_{L}^{+}[16,17]$ que viola a unitariedade à altas energias sem 
o Higgs. Os diagramas para esse processo estão ilustrados na figura (3.2). Podemos calcular a amplitude para esse espalhamento usando o teorema da equivalência dos bósons de Goldstone [18] e em seguida aplicando a condição de unitariedade do formalismo de ondas parciais. Esse teorema diz que:

"A altas energias, a amplitude para emissão ou absorção de um bóson de gauge massivo longitudinalmente polarizado vem a ser igual à amplitude para emissão ou absorção do bóson de Goldstone que foi absorvido pelo bóson de gauge"

$$
\mathcal{M}\left(W_{L}^{ \pm}, Z_{L}^{0}\right)=\mathcal{M}\left(\omega^{ \pm}, z^{0}\right)+\mathcal{O}\left(\frac{M_{W, Z}}{\sqrt{s}}\right)
$$

para $s \gg M_{W}^{2}, M_{Z}^{2}$. Antes de aplicar o teorema devemos escrever a lagrangeana de interação do Higgs com os bósons de Goldstone,

$$
\mathcal{L}=\left(\partial_{\mu} \Phi\right)^{\dagger}\left(\partial^{\mu} \Phi\right)+\mu^{2} \Phi^{\dagger} \Phi-\lambda\left(\Phi^{\dagger} \Phi\right)^{2},
$$

com $V=-\mu^{2} \Phi^{\dagger} \Phi+\lambda\left(\Phi^{\dagger} \Phi\right)^{2}$. Podemos escrever o dubleto do Higgs em termos de $\omega^{ \pm}, z^{0}(2.81)$,

$$
\Phi=\frac{1}{\sqrt{2}}\left(\begin{array}{c}
i \sqrt{2} \omega^{+} \\
v+h-i z^{0}
\end{array}\right)=\left(\begin{array}{c}
\phi^{+} \\
\phi^{0}
\end{array}\right) .
$$

O termo de interação da lagrangeana fica da seguinte forma

$$
\begin{gathered}
\mathcal{L}_{\text {int }}=-\lambda v h\left(2 \omega^{+} \omega^{-}+z^{2}+h^{2}\right)-\frac{1}{4} \lambda\left(2 \omega^{+} \omega^{-}+z^{2}+h^{2}\right)^{2} \\
=-\frac{g}{4} \frac{M_{H}^{2}}{M_{W}} h\left(h^{2}+2 \omega^{+} \omega^{-}+z^{2}\right)-\frac{g^{2}}{32} \frac{M_{H}^{2}}{M_{W}^{2}}\left(h^{2}+2 \omega^{+} \omega^{-}+z^{2}\right)^{2},
\end{gathered}
$$

onde $\lambda=\frac{G_{F} M_{H}^{2}}{\sqrt{2}}=\frac{g^{2}}{8} \frac{M_{H}^{2}}{M_{W}^{2}}$. Usando o teorema da equivalência a amplitude fica 


$$
\begin{gathered}
\mathcal{M}\left(W_{L}^{+} W_{L}^{-} \rightarrow W_{L}^{+} W_{L}^{-}\right) \simeq \mathcal{M}\left(\omega^{+} \omega^{-} \rightarrow \omega^{+} \omega^{-}\right)=-i \frac{g^{2}}{4} \frac{M_{H}^{2}}{M_{W}^{2}} \\
\times\left(\frac{s}{s-M_{H}^{2}}+\frac{t}{t-M_{H}^{2}}\right)=-i \frac{g^{2}}{4} \frac{M_{H}^{2}}{M_{W}^{2}}\left(2+\frac{M_{H}^{2}}{s-M_{H}^{2}}+\frac{M_{H}^{2}}{t-M_{H}^{2}}\right) .
\end{gathered}
$$

No limite de altas energias $\left(s \gg M_{H}^{2}\right)$ encontramos

$$
\mathcal{M}=-i \frac{g^{2}}{2} \frac{M_{H}^{2}}{M_{W}^{2}}=-i 2 \sqrt{2} G_{F} M_{H}^{2} .
$$

Utilizando a expansão em ondas parciais a amplitude pode ser escrita na seguinte forma

$$
\mathcal{M}=16 \pi \sum_{J}(2 J+1) a_{J} P_{J}(\cos \theta) .
$$

A condição de unitariedade para $J=0$ impõe que

$$
a_{0} \leq 1, \quad a_{0}=\frac{\mathcal{M}}{16 \pi},
$$

logo, o limite superior para a massa do Higgs pode ser encontrado,

$$
\begin{gathered}
\frac{G_{F} M_{H}^{2}}{4 \pi \sqrt{2}} \leq 1, \\
M_{H} \leq \frac{4 \pi \sqrt{2}}{G_{F}} \simeq 1 T e V
\end{gathered}
$$




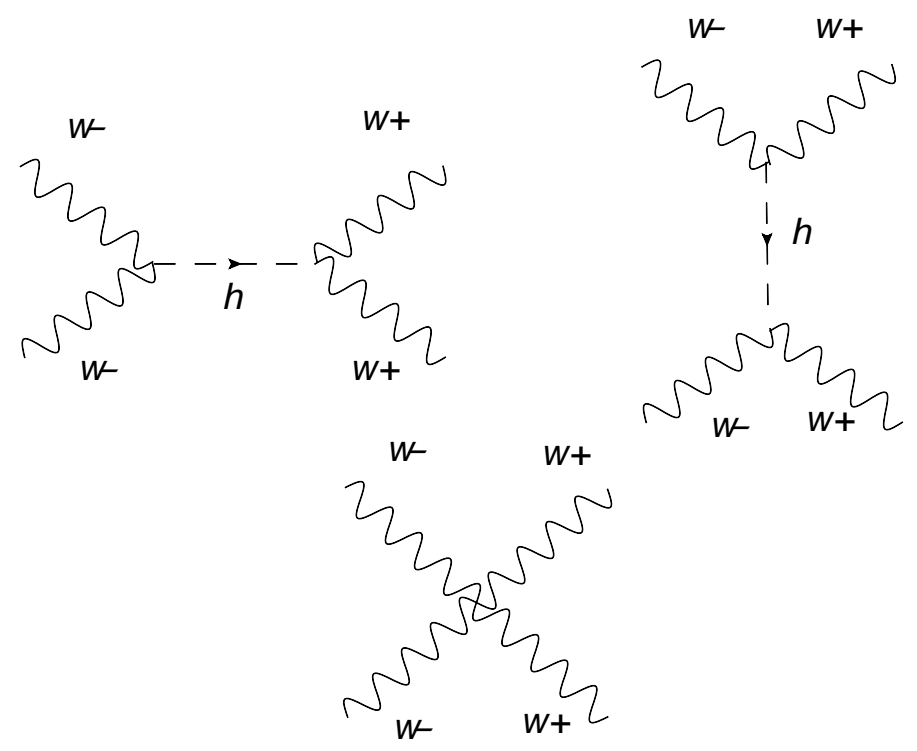

Figura 3.2. Diagramas de Feynman para o espalhamento $W^{+} W^{-} \rightarrow W^{+} W^{-}$.

\subsubsection{O Problema da Massa dos Férmions}

Outro problema de hierarquia fundamental no MP é o que diz respeito à massa dos férmions. Como vimos nas equações $(2.89,2.106,2.107)$ os termos de massa fermiônicos podem ser escritos na seguinte forma

$$
m_{f}=\frac{G_{f} v}{\sqrt{2}}
$$

onde $G_{f}$ são os acoplamentos de Yukawa dos férmions. O problema é que esses acoplamentos são parâmetros livres na teoria (12 no total), sendo ajustados de acordo com os valores observados experimentalmente para a massa de cada férmion. Para o quark up por exemplo, temos $G_{f} \sim 10^{-5}$, para o quark charm $G_{f} \sim 10^{-2}$ e para o quark top $G_{f} \sim 1$. Sendo assim, o Modelo Padrão não explica porque partículas com os mesmos números quânticos (carga, hipercarga, isospin) possuem massas tão diferentes. Já que as massas dessas partículas são geradas da mesma maneira (mecanismo de Higgs), o mais natural seria que as massas fossem iguais ou da mesma ordem de grandeza. 


\subsubsection{Outros Problemas ${ }^{1}$}

Um outro problema de hierarquia no MP é o que se refere a constante cosmológica, pois o valor esperado teoricamente para a densidade de energia do vácuo - relacionada com a constante cosmológica pela equação $\Lambda=8 \pi G \rho_{\text {vác.- }}$ é 120 ordens de grandeza maior do que o observado experimentalmente.

Um outro componente do universo, tão importante quanto a energia do vácuo (energia escura), é a matéria escura. Conhecida desde a década de 30, a natureza dessa matéria, que interage apenas gravitacionalmente com a matéria comum (bariônica), permanece um mistério. Muitas teorias físicas além do MP (Supersimetria e UED, Universal Extra Dimensions) propõem novas partículas, das quais seria composta a matéria escura.

Sabemos que as interações fracas violam a simetria CP (conjugação de carga e paridade), que é uma simetria que transforma uma partícula de mão esquerda, por exemplo, em sua anti-partícula de mão direita; no entanto, a violação dessa simetria não é observada nas interações fortes, o que é um problema já que na Lagrangiana da QCD existe um termo que viola a simetria CP e cujo coeficiente é experimentalmente vinculado a ser menor que $\sim 10^{-10}$, o que requer uma simetria para ser explicado [20]. A solução desse problema poderia ajudar na elucidação de um outro problema, o da assimetria matéria-antimatéria. Sabemos que razão entre as densidades do número de bárions e do número de fótons é de uma em 1 bilhão, significando que para cada $10^{9}$ anti-partículas foram criadas $10^{9}+1$ partículas, quando o mais natural seria que partículas e anti-partículas fossem criadas na mesma quantidade.

Outro problema relacionado com o problema da hierarquia é a origem da quebra da simetria eletrofraca, que discutiremos no próximo capítulo.

\footnotetext{
${ }^{1}$ Uma discussão mais aprofundada desses problemas pode ser vista na referência [19].
} 


\subsection{Teorias além do Modelo Padrão}

\subsubsection{Teorias com Dimensões Extras}

A idéia de construir teorias com dimensões extras não é nova, data do início do século XX, quando os matemáticos Theodor Kaluza e Oskar Klein propuseram dimensões extras compactas com o objetivo de unificar as duas forças conhecidas na época, o eletromagnetismo e a gravitação de Einstein (teoria recém criada na época, 1915). Com a descoberta de novas interações (fraca e forte) nas décadas seguintes, a idéia de Kaluza-Klein foi ressucitada com o objetivo de unificar todas as interações utilizando dimensões extras, nas chamadas Teorias de Cordas e Supercordas [19, 21, 22].

\section{LED (Large Extra Dimensions)}

A primeira teoria com dimensões extras direcionada a resolver os problemas do Modelo Padrão foi a Teoria com Dimensões Extras Compactas (LED), proposta no final da década de 90 por Arkani-Hamed, S. Dimopoulos e G. Divali [23], com o objetivo de resolver o problema da hierarquia. Nesse modelo com $\mathrm{N}$ dimensões a gravidade é diluída pelo volume das dimensões extras, já que é a única interação livre para se propagar nessas dimensões (as interações eletromagnética, fraca e forte continuam limitadas a se propagar nas quatro dimensões do espaço-tempo). A ação de Einstein-Hilbert nesse espaço N-dimensional tem a forma

$$
S=-\frac{1}{16 \pi G_{*}} \int d^{4+n} x \sqrt{\left|g_{(4+n)}\right|} R_{(4+n)},
$$

onde $G_{*}$ é a constante de acoplamento gravitacional fundamental, $g_{(4+n)}$ é o determinante da métrica do espaço com $(4+n)$ dimensões, e $R_{(4+n)}$ é o tensor de Ricci nesse espaço. Para obtermos a constante $G_{*}$ vamos assumir a seguinte métrica com dimensões extras planas,

$$
d s^{2}=\eta_{\mu \nu} d x^{\mu} d x^{\nu}-\delta_{a b} d y^{a} d y^{b}
$$


onde $\eta_{\mu \nu}$ é a métrica 4-dimensional, e $\delta_{a b} d y^{a} d y^{b}$ nos dá os elementos da dimensão extra. Com essa métrica temos que $\sqrt{\mid g_{(4+n) \mid}}=\sqrt{\left|g_{(4)}\right|}$ e $R_{(4+n)}=R_{(4)}$, e a integração nas dimensões extras nos dá a ação efetiva 4-dimensional,

$$
S=-\frac{V_{n}}{16 \pi G_{*}} \int d^{4} x \sqrt{\left|g_{(4)}\right|} R_{(4)},
$$

onde $V_{n}=R^{n}$ é o volume do espaço ocupado pelas dimensões extras e $R$ é o raio de compactificação dessas dimensões. Comparando essa ação com a ação em 4D que conhecemos, encontramos a relação

$$
G_{N}=\frac{G_{*}}{V_{n}}
$$

onde $G_{N}$ é a constante gravitacional de Newton (a constante efetiva da nossa teoria), e está suprimida pelo volume do espaço com $n$ dimensões extras. Como a escala de Planck é definida em termos da constante de Newton pela relação

$$
M_{P} c^{2}=\left[\frac{\hbar c^{5}}{8 \pi G_{N}}\right]^{1 / 2},
$$

e sendo $M_{*}$ a escala fundamental da nossa teoria com $n$ dimensões extras, então

$$
M_{*} c^{2}=\left[\frac{\hbar^{1+n} c^{5+n}}{8 \pi G_{*}}\right]^{1 /(2+n)} .
$$

A escala de Planck é suprimida e a escala fundamental da gravidade é a escala $M_{*}$. Utilizando o sistema natural de unidades obtemos a seguinte relação entre as duas escalas,

$$
M_{P}^{2}=M_{*}^{n+2} R^{n},
$$

Para resolver o problema da hierarquia precisamos que $M_{*} \simeq 1 \mathrm{TeV}$, logo

$$
R \sim 2 \times 10^{-17+\frac{32}{n}} \mathrm{~cm} .
$$


Para $n=1, R \sim 10^{11} m$, já empiricamente excluído, enquanto que os limites para $n=2$ já são cobertos pelos atuais experimentos.

A conservação do momento 5-dimensional $p_{M}^{2}=0$ implica em

$$
p_{\mu}^{2}-\frac{N^{2}}{R^{2}}=0
$$

$\operatorname{com} p_{5}=\frac{N}{R}$ e $m_{N}^{2}=m^{2}+\frac{N^{2}}{R^{2}}$, onde $\mathrm{N}$ são os modos de Kaluza-Klein, e $m=0$ é a massa do gráviton. Temos então novos estados de spin 2, excitações de KK do gráviton, com gap de massa entre os modos de $\triangle m \sim 1 / R$. Para $n=2$, a massa do primeiro estado excitado do gráviton é $m_{1} \sim 10^{-3} \mathrm{eV}$, e com $n=3, m_{1} \sim 100 \mathrm{eV}$. Sinais para a detecção do gráviton seriam obtidos através de processos com grande energia transversa perdida (MET, missing energy), pois o tempo de vida dessas partículas é suprimido pela $M_{P}$.

\section{UED (Universal Extra Dimensions)}

Nesse caso [24, 25], além do campo gravitacional, todos os outros campos estão livres para se propagar no bulk ${ }^{2}$. Isso gera vínculos maiores em $1 / R$, já que os acoplamentos desses campos não são suprimidos gravitacionalmente. A compactificação, no caso $5 \mathrm{D}$ sob a simetria $S_{1} / Z_{2}{ }^{3}$, leva a conservação do momento 5-dimensional e consequentemente a conservação do número de KK, gerando vínculos da ordem $1 / R \sim 300 \mathrm{GeV}$. A conservação do número de Kaluza-Klein implica, entre outras coisas, que os modos de KK só podem ser produzidos em pares, proibindo processos em que os modos zero (partículas do MP) se combinem para produzir um bóson excitado de KK. Esse fato contribui para que o estado excitado mais leve de KK, que não pode decair em modos zero seja a partícula candidata à matéria escura.

\footnotetext{
${ }^{2} \mathrm{O}$ espaço 5 -dimensional.

${ }^{3} S_{1}$ é a esfera de dimensão 1 e $Z_{2}$ a simetria que identifica os pontos y com os -y na coordenada da dimensão extra
} 


\subsubsection{Teorias com Dimensões Extras Curvas (WED)}

Diferente das propostas anteriores (LED e UED), onde a métrica era plana, esse modelo proposto por Lisa Randall e Raman Sundrum [26], propõe uma dimensão extra curva com uma métrica anti-de Sitter (AdS) em cinco dimensões para resolver o problema da hierarquia. Essa métrica tem a seguinte forma

$$
\begin{gathered}
d s^{2}=g_{M N} d x^{M} d x^{N}, \\
d s^{2}=e^{-2 k y} \eta^{\mu \nu} d x_{\mu} d x_{\nu}-d y^{2},
\end{gathered}
$$

onde

$$
g_{M N}=\left(\begin{array}{cc}
g_{\mu \nu} & 0 \\
0 & -1
\end{array}\right)=\left(\begin{array}{cc}
e^{-2 k y} \eta_{\mu \nu} & 0 \\
0 & -1
\end{array}\right),
$$

obtida pela solução das equações de Einstein em 5D, sendo y a coordenada da dimensão extra e k a curvatura AdS do espaço-tempo. Podemos interpretar essa métrica como descrevendo uma soma de infinitas fatias 4-dimensionais (branas) ao longo de toda dimensão extra (y). A dimensão extra é compactificada no orbifold $S_{1} / Z_{2}$, sendo $S_{1}$ a esfera de dimensão 1 (circunferência) e raio $R$, e $Z_{2}$ a simetria (necessária para que os férmions sejam quirais) que identifica os pontos y com os -y na coordenada da quinta dimensão. Com essa compactificação podemos dividir $S_{1}$ em dois círculos com a coordenada y variando de 0 até $\pi R$.

Para resolver o problema da hierarquia nesse modelo, precisamos relacionar duas escalas de energia distintas, as escalas de Planck e TeV, separadas 16 ordens de grandeza uma da outra. Podemos fazer isso localizando cada uma delas em branas diferentes ao longo da dimensão extra. A diferença entre uma grandeza medida em branas distintas será dada pelo fator exponencial da métrica (3.17). Localizando a escala de Planck na brana em $y=0$ e a escala eletrofraca em $y=\pi R$, 


$$
M_{e}=\Lambda_{e}=M_{P} e^{-k \pi R}
$$

com $k R \simeq 12$ para resolver o problema da hierarquia.

Para entender melhor essa supressão da escala de Planck (que nesse caso é a escala fundamental) pelo fator exponencial da métrica AdS vamos usar o campo do Higgs, localizado na brana em $\pi R$, como exemplo. A ação do Higgs tem a seguinte forma

$$
S_{H}=\int d^{4} x \int_{0}^{\pi R} d y \sqrt{-g} \delta(y-\pi R)\left[g^{\mu \nu} \partial_{\mu} H^{\dagger} \partial_{\nu} H-\lambda\left(|H|^{2}-v_{0}^{2}\right)^{2}\right]
$$

onde

$$
g^{\mu \nu}(x, \pi R)=e^{2 k \pi R} \eta^{\mu \nu} \quad e \quad \sqrt{-g}=e^{-4 k y},
$$

Substituindo na ação do Higgs e integrando em y, obtemos

$$
S_{H}=\int d^{4} x\left[e^{-2 k \pi R} \eta^{\mu \nu} \partial_{\mu} H^{\dagger} \partial_{\nu} H-e^{-4 k \pi R} \lambda\left(|H|^{2}-v_{0}^{2}\right)^{2}\right] .
$$

Depois da renormalização da função de onda, $H \rightarrow e^{k \pi R} H$, encontramos

$$
S_{H}=\int d^{4} x\left[\eta^{\mu \nu} \partial_{\mu} H^{\dagger} \partial_{\nu} H-\lambda\left(|H|^{2}-e^{-2 k \pi R} v_{0}^{2}\right)^{2}\right],
$$

sendo o VEV efetivo dado por

$$
v \equiv e^{-k \pi R} v_{0}
$$

que para $k R \sim 12$, com $v_{0} \sim k \sim M_{P}$, restaura a escala eletrofraca $v \simeq$ $246 \mathrm{GeV}$, desde que o Higgs esteja localizado próximo à brana $\mathrm{TeV}$. 


\section{Modelo Padrão no bulk}

No modelo de Randall-Sundrum somente a gravidade está livre para se propagar pela dimensão extra. Embora resolva o problema da hierarquia, isso apresenta alguns problemas, principalmente associado ao fato de não suprimir operadores de dimensão maior na escala de Planck, consequentemente isso traz problemas (não suprime) para o decaimento do próton [27]. Alguns desses problemas podem ser resolvidos deixando os férmions e os bósons de gauge livres para se propagar no bulk, enquanto o Higgs permanece localizado na brana $\mathrm{TeV}[28,29,30,31,32,33,34]$. Isso traz importantes consequências para a teoria, como a possibilidade de explicar a hierarquia fermiônica, e principalmente a ferramenta para construir modelos da quebra da simetria eletrofraca (EWSB) [3].

A ação 5-dimensional para os bósons de gauge, férmions e escalares no bulk é dada por

$$
-\int d^{4} x \int d y \sqrt{-g}\left[\frac{1}{4 g_{5}} F_{M N}^{2}+\left|\partial_{M} \phi\right|^{2}+m_{\phi}^{2}|\phi|^{2}+i \bar{\psi} \gamma^{M} D_{M} \psi+m_{\psi} \bar{\psi} \psi\right]
$$

onde $F_{M N}=\partial_{M} A_{N}-\partial_{N} A_{M}$ é o tensor do campo eletromagnético em cinco dimensões, $D_{M}=\partial_{M}+\Gamma_{M}$ é a derivada covariante no espaço curvo $5 \mathrm{D}$, sendo $\Gamma_{\mu}$ a spin connection, que para a métrica $A d S_{5}$ (3.17) tem a forma

$$
\Gamma_{\mu}=\frac{1}{2} \gamma_{5} \gamma_{\mu} \frac{d \sigma}{d y} \quad \text { e } \quad \Gamma_{5}=0
$$

onde $\sigma=k y$, e as matrizes $\gamma_{M}=\left(\gamma_{\mu}, \gamma_{5}\right)$ são definidas no espaço-tempo curvo como $\gamma_{M}=e_{M}^{\alpha} \gamma_{\alpha}$, sendo $e_{M}^{\alpha}$ o vierbein e $\gamma_{\alpha}$ as matrices de Dirac no espaço plano.

Expandindo os campos 5-dimensionais,

$$
\Phi\left(x^{\mu}, y\right)=\frac{1}{\sqrt{2 \pi R}} \sum_{n=0}^{\infty} \Phi^{(n)}\left(x^{\mu}\right) f_{n}(y)
$$


com a função $f_{n}(y)$ obedecendo a condição de ortonormalização,

$$
\frac{1}{2 \pi R} \int_{-\pi R}^{\pi R} d y e^{(2-s)} f_{n}(y) f_{m}(y)=\delta_{n m},
$$

onde $\Phi=\left\{A_{\mu}, \phi, e^{-2 \sigma} \psi_{L, R}\right\}, s=\{2,4,1\}$. Substituindo as expressões (3.26, 3.27) na ação 5D (3.24) impondo as condições de contorno de Dirichlet e Neumann para os campos 5-dimensionais,

$$
\left\{\begin{array}{c}
\phi\left(x^{\mu}, y\right),\left.A_{5}\left(x^{\mu}, y\right)\right|_{y=0, \pi R}=0 \\
\partial_{5} \phi\left(x^{\mu}, y\right),\left.\partial_{5} A_{\mu}\left(x^{\mu}, y\right)\right|_{y=0, \pi R}=0
\end{array}\right.
$$

e finalmente integrando em y, obtemos que as funções $f_{n}(y)$ devem satifazer a equação

$$
\left[-e^{s \sigma} \partial_{5}\left(e^{-s \sigma} \partial_{5}\right)+M_{\Phi}^{2}\right] f_{n}(y)=e^{2 \sigma} m_{n}^{2} f_{n}(y),
$$

onde definimos o quadrado do parâmetro de massa como $M_{\Phi}^{2}=\left\{0, a k^{2}, c(c \pm 1) k^{2}\right\} .^{4}$

\section{Exemplo (bósons de gauge):}

Vamos obter a equação (3.29) para um exemplo em particular, os bósons de gauge se propagando pelo bulk [29, 30, 32]. Nesse caso, a ação pode ser escrita como

$$
\begin{gathered}
S_{G}=-\frac{1}{4 g_{5}} \int d^{4} x d y \sqrt{-g} g^{M N} g^{K L} F_{M K} F_{N L} \\
=\int d^{4} x d y\left[\frac{e^{-4 \sigma}}{g_{5}}\right]\left\{-\frac{e^{4 \sigma}}{4} F_{\mu \nu} F^{\mu \nu}+e^{2 \sigma}\left[\frac{1}{2}\left(\partial_{5} A_{\mu}\right)^{2}-\partial_{5} A_{\mu} \partial^{\mu} A_{5}+\left(\partial_{\mu} A_{5}\right)^{2}\right]\right\} .
\end{gathered}
$$

Para simplificar, estamos usando o gauge $A_{5}=0$ e impondo o vínculo da lei de Gauss $\partial_{5}\left(\partial_{\mu} A_{5}\right) \approx 0$. Após uma integração por partes, obtemos

\footnotetext{
${ }^{4} \mathrm{~A}$ parametrização usada aqui é: $m_{\phi}^{2}=a k^{2}+b \sigma^{\prime \prime}, m_{\Psi}=c \sigma^{\prime}$, onde $\sigma^{\prime}=k \epsilon(y)$ e $\sigma^{\prime \prime}=2 k[\delta(y)-\delta(y-\pi R)]$, sendo que não estamos considerandos os termos proporcionais a $\sigma^{\prime \prime}[27]$.
} 


$$
=-\frac{1}{4 g_{5}} \int d^{4} x d y\left\{F_{\mu \nu} F^{\mu \nu}+2 A_{\mu} \partial_{5}\left(e^{-2 \sigma} \partial_{5} A^{\mu}\right)\right\},
$$

onde usamos as condições de contorno (3.28) para campo de gauge. Agora, expandindo o campo $A_{\mu}$ usando (3.26),

$$
A_{M}\left(x^{\mu}, y\right)=\frac{1}{\sqrt{2 \pi R}} \sum_{n=0}^{\infty} A_{\mu}^{(n)}\left(x^{\mu}\right) \chi_{n}(y)
$$

encontramos

$$
=\sum_{n} \int d^{4} x d y \frac{1}{g_{5}}\left\{-\frac{1}{4} F_{\mu \nu}^{(n)} F^{(m) \mu \nu} \chi_{n} \chi_{m}+\frac{1}{2} A_{\mu}^{(n)} A^{(m) \mu} \chi_{n} \partial_{5}\left(e^{-2 \sigma} \partial_{5} \chi_{n}\right)\right\} .
$$

Integrando na coordenada da dimensão extra, utilizando a condição de ortonormalização (3.27) para $\chi_{n}(s=2)$, e impondo que essa ação seja igual a ação para o campo de gauge em $4 \mathrm{D}$,

$$
S_{G}^{4 D}=\sum_{n} \int d^{4} x \frac{1}{g_{5}}\left\{-\frac{1}{4} F_{\mu \nu}^{(n)} F^{(n) \mu \nu}-\frac{1}{2} m_{n}^{2} A_{\mu}^{(n)} A^{(n) \mu}\right\},
$$

encontramos que a seguinte equação deve ser satisfeita

$$
-\partial_{5}\left(e^{-2 \sigma} \partial_{5} \chi_{n}(y)\right)=m_{n}^{2} \chi_{n}(y)
$$

que é a mesma equação (3.29) $\operatorname{com} s=2$.

Para os férmions quirais definidos como $\psi_{L, R}= \pm \gamma_{5} \psi_{L, R}$, encontramos que as funções $f_{n}^{R}$ e $f_{n}^{L}$ devem satisfazer o seguinte sistema de equações diferenciais acopladas:

$$
\left\{\begin{array}{c}
\left(\partial_{5}-m_{\psi}\right) f_{n}^{R}(y)=-m_{n} e^{\sigma} f_{n}^{L}(y) \\
\left(\partial_{5}+m_{\psi}\right) f_{n}^{L}(y)=m_{n} e^{\sigma} f_{n}^{R}(y)
\end{array}\right.
$$

Voltando à equação (3.29), a solução geral é dada por 


$$
f_{n}(y)=\frac{e^{s \sigma / 2}}{N_{n}}\left[J_{\alpha}\left(\frac{m_{n}}{k} e^{\sigma}\right)+b_{\alpha}\left(m_{n}\right) Y_{\alpha}\left(\frac{m_{n}}{k} e^{\sigma}\right)\right],
$$

onde $m_{n}$ são as massas de KK e $J_{\alpha}$ e $Y_{\alpha}$ são funções de Bessel de ordem $\alpha=\sqrt{\left(\frac{s}{2}\right)^{2}+\frac{M_{\Phi}^{2}}{k^{2}}}, b_{\alpha}$ é uma constante e $N_{n}$ é o fator de normalização, que pode ser encontrado usando a condição de ortonormalização (3.27),

$$
N_{n}^{2}=\frac{1}{\pi R} \int_{0}^{\pi R} d y e^{2 \sigma}\left[J_{\alpha}\left(\frac{m_{n}}{k} e^{\sigma}\right)+b_{\alpha}\left(m_{n}\right) Y_{\alpha}\left(\frac{m_{n}}{k} e^{\sigma}\right)\right]^{2} .
$$

Os coeficientes $b_{\alpha}\left(m_{n}\right)$ e as massas de Kaluza-Klein são determinados impondo condições de contorno nas soluções para $f_{n}$, dependendo também se o campo é ímpar ou par sob a simetria $Z_{2}$.

\section{Campos pares:}

Nesse caso a função $f_{n}$ deve satisfazer às seguintes condições:

$$
\begin{gathered}
f_{n}(y) \rightarrow f_{n}(|y|), \\
\left.\left(\frac{d f_{n}}{d y}-r \sigma^{\prime} f_{n}\right)\right|_{0, \pi R}=0,
\end{gathered}
$$

onde $r=\{0, b, \mp c\}$ para $\Phi=\left\{A_{\mu}, \phi, e^{-2 \sigma} \psi_{L, R}\right\}$. Impondo essas condições na solução geral para $f_{n}$, encontramos

$$
\begin{gathered}
b_{\alpha}\left(m_{n}\right)=-\frac{\left(-r+\frac{s}{2}\right) J_{\alpha}\left(\frac{m_{n}}{k}\right)+\frac{m_{n}}{k} J_{\alpha}^{\prime}\left(\frac{m_{n}}{k}\right)}{\left(-r+\frac{s}{2}\right) Y_{\alpha}\left(\frac{m_{n}}{k}\right)+\frac{m_{n}}{k} Y_{\alpha}^{\prime}\left(\frac{m_{n}}{k}\right)}, \\
b_{\alpha}\left(m_{n}\right)=b_{\alpha}\left(m_{n} e^{\pi k R}\right) .
\end{gathered}
$$

No limite $m_{n} \ll k$ e $k R \gg 1$ obtemos para as massas de KK

$$
m_{n} \simeq\left(n+\frac{\alpha}{2}-\frac{3}{4}\right) \pi k e^{-\pi k R}
$$

para $n=1,2,3, \ldots$ e $\alpha>0$. 


\section{Campos ímpares:}

Para campos ímpares sob a simetria $Z_{2}$ temos

$$
\begin{gathered}
f_{n}(y) \rightarrow-f(|y|), \\
\left.f_{n}\right|_{0, \pi R}=0 .
\end{gathered}
$$

Aplicando essas condições na solução geral (3.31) é fácil ver que

$$
\begin{gathered}
b_{\alpha}\left(m_{n}\right)=-\frac{J_{\alpha}\left(\frac{m_{n}}{k}\right)}{Y_{\alpha}\left(\frac{m_{n}}{k}\right)}, \\
b_{\alpha}\left(m_{n}\right)=b_{\alpha}\left(m_{n} e^{\pi k R}\right) .
\end{gathered}
$$

No limite $m_{n} \ll k$ e $k R \gg 1$, encontramos

$$
m_{n} \simeq\left(n+\frac{\alpha}{2}-\frac{1}{4}\right) \pi k e^{-\pi k R}
$$

com $n=1,2,3, \ldots$ e $\alpha>0$. Comparando as soluções de $m_{n}$ vemos que a diferença de massa entre os modos pares e ímpares é de $\pi / 2$, sendo que o gap de massa entre dois modos consecutivos é $\triangle m \simeq \pi k e^{-\pi k R}$.

Vamos agora analisar o comportamento da função $f_{n}(y)$ (3.31). No caso do campo escalar $(s=4), f_{n}$ aumenta com y para os primeiros modos de $\mathrm{KK}$, sendo as soluções para esses modos localizadas na brana em $y=\pi R$, resultando em fortes acoplamentos com campos localizados na brana $\mathrm{TeV}$ e acoplamentos fracos com campos localizados na brana de Planck. Para os bósons de gauge $(s=2), f_{n}$ também está localizada próxima à brana de $\mathrm{TeV}$, se acoplando fortemente com os campos localizados nessa brana (fig. 3.1). Se colocarmos somente os bósons de gauge para se propagar no bulk, vínculos indicam que a massa do primeiro bóson de gauge excitado deveria ser maior que $23 \mathrm{TeV}$ [29]. Na referência [27] foi mostrado que esse limite pode ser relaxado para $m_{1} \gtrsim 2.1\left(g_{1} / g\right) T e V$ quando os férmions também são livres 
para se propagar pelo bulk, sendo que a razão $g_{1} / g$ ( $g_{1}$ é o acoplamento do bóson de KK com os férmions do MP-a ser discutido no próximo capítulo- e g é o acoplamento do MP) depende do parâmetro c de localização dos férmions na coordenada da dimensão extra.

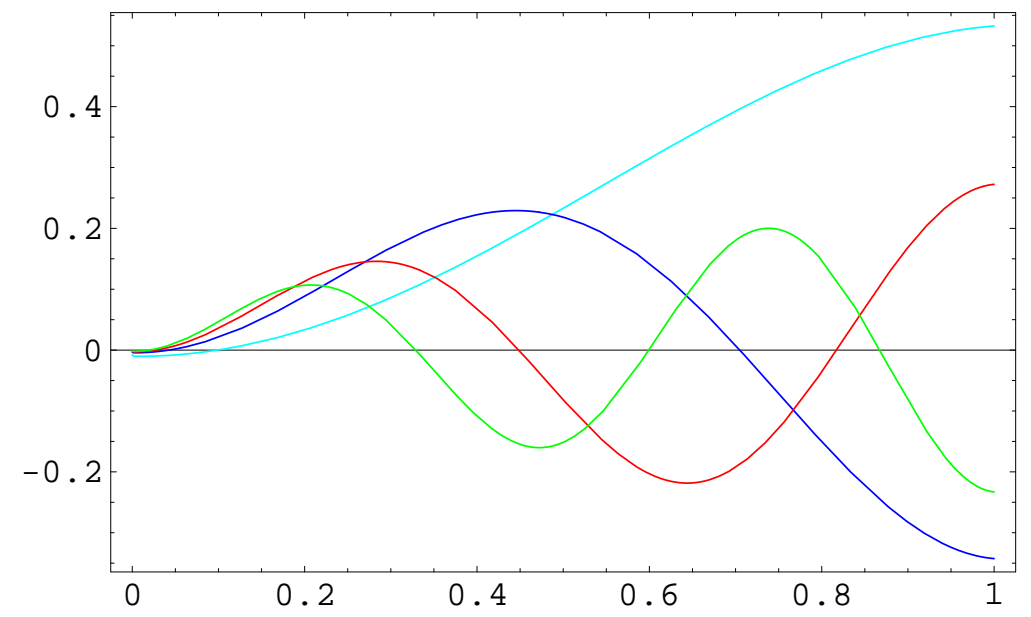

Figura 3.1: Comportamento da função de onda $f_{n}(y)$ para diferentes modos dos bósons de KK. Linha azul claro $(\mathrm{n}=1)$, linha azul $(n=2)$, linha vermelha $(n=3)$, linha verde $(n=4)$.

\section{Hierarquia fermiônica}

Podemos também encontrar soluções de $f_{n}$ para os férmions do Modelo Padrão, os modos zero de $f_{n}$. Nesse caso $m_{0}=0$, então as equações (3.30) ficam

$$
\left\{\begin{array}{l}
\left(\partial_{5}-m_{\psi}\right) f_{0}^{R}(y)=0 \\
\left(\partial_{5}+m_{\psi}\right) f_{0}^{L}(y)=0
\end{array}\right.
$$

As soluções têm a forma

$$
f_{0}^{R, L}(y)=N_{0} e^{ \pm c k y}
$$


sendo que o coeficiente $N_{0}$ pode ser obtido utilizando a condição de ortonormalização para as funções $f_{n}(3.27)$, então

$$
f_{0}^{R, L}(y)=\sqrt{\frac{(1 \pm 2 c) k \pi R}{e^{(1 \pm 2 c) k \pi R}-1}} e^{ \pm c k y}
$$

Esse resultado mostra que $f_{0}^{R, L}$ tem uma dependência com o parâmetro c (parâmetro de massa dos férmions), que indica como ela se comporta próxima das branas de Planck e TeV, e como os campos fermiônicos podem se acoplar com os campos localizados nessas branas. Podemos ver melhor isso olhando para os acoplamentos de Yukawa dos férmions com o Higgs, confinado na brana TeV. A ação que descreve essa interação pode ser escrita como

$$
\int d^{4} x \int d y \sqrt{-g} \lambda_{i j}^{(5)} H(x)\left(\bar{\psi}_{i L}(x, y) \psi_{j R}(x, y)+\text { h.c. }\right) \delta(y-\pi R)
$$

onde $\lambda_{i j}^{(5)}$ são os acoplamentos de Yukawa 5-dimensionais e i e j são os índices de sabor dos férmions. Expandindo os campos fermiônicos, usando a relação $(3.26)$,

$$
\psi\left(x^{\mu}, y\right)=\frac{1}{\sqrt{2 \pi R}} \psi^{(0)}\left(x^{\mu}\right) f_{0}(y)=\frac{e^{(2-c) k y}}{\sqrt{2 \pi R} N_{0}} \psi_{R, L}^{(0)}(x) .
$$

Substituindo $\psi\left(x^{\mu}, y\right)$ na ação e integrando em y, com $\sqrt{-g}=e^{-4 k y}$ e o campo do Higgs canonicamente normalizado $H(x) \rightarrow e^{k \pi R} H(x)$, encontramos a ação efetiva 4-dimensional,

$$
S_{Y u k}=\int d^{4} x \lambda_{i j} H(x)\left(\bar{\psi}_{i L}^{(0)}(x) \psi_{j R}^{(0)}(x)+\text { h.c. }+\ldots\right),
$$

onde $\lambda_{i j}$ são os acoplamentos 4D de Yukawa dos férmions de modo zero (férmions do MP), $\psi_{i L}^{(0)}$ e $\psi_{j R}^{(0)}$,

$$
\lambda_{i j}=\frac{\lambda_{i j}^{(5)} k}{N_{i L} N_{j R}} e^{\left(1-c_{i L}-c_{i R}\right) \pi k R},
$$

onde 


$$
N_{i L}^{2}=\frac{e^{\left(1-2 c_{i L}\right) \pi k R}-1}{1 / 2-c_{i L}} .
$$

Dessa forma podemos ver que diferentes valores de c resultam em diferentes acoplamentos de Yukawa, gerando diferentes massas para os férmions. Sendo assim, a massa dos férmions leves, fracamente acoplados com o Higgs, podem ser geradas naturalmente impondo valores de c que localizem suas funções de onda na brana de Planck, enquanto os férmions pesados $(c, \tau, b, t)$ possuem acoplamentos maiores com o Higgs, não podendo ser confinados próximos à brana de Planck ${ }^{5}$. Assim, o espectro de massas fermiônico é explicado naturalmente, de acordo com a localização dos modos zero de cada férmion na dimensão extra.

\footnotetext{
${ }^{5} \mathrm{O}$ top com um grande acoplamento de Yukawa $\lambda \sim 1$ deve ser localizado na brana $\mathrm{TeV}$.
} 


\section{Capítulo 4}

\section{Quebra da Simetria Eletrofraca}

Nesse capítulo vamos estudar e analisar a fenomenologia do modelo proposto por G. Burdman e L. da Rold [5], que atribui à quebra da simetria eletrofraca a condensação de uma quarta geração de férmions. Barden, Hill e Lindner [35] já haviam proposto a condensação do Top como origem dinâmica da EWSB, mas como veremos adiante a nova dinâmica estaria presente na escala $\Lambda \sim 10^{15} \mathrm{GeV}$ para $m_{t} \simeq 175 \mathrm{GeV}$. Isso não resolve o problema da hierarquia, pois para restaurar a escala $\mathrm{TeV}$ precisamos de uma partícula que condense com massa entre $(600-700) \mathrm{GeV}$. Essa é a motivação para propor a condensação de uma quarta geração de quarks como origem dinâmica da EWSB. No entanto, isso não é suficiente para explicar a massa de todos os outros férmions do Modelo Padrão que não condensam. Em teorias com uma dimensão extra curva (WED) esse problema é explicado naturalmente permitindo que os campos fermiônicos se propaguem pela dimensão extra, e relacionando a massa de cada férmion com um parâmetro que dá a sua localização no bulk. Podemos perguntar então, é possível construir uma teoria fracamente acoplada no espaço 5D que descreva uma teoria fortemente acoplada em 4D? Nossa resposta é sim, isso é possível de acordo com a correspondência AdS/CFT [4, 36, 37]. 


\subsection{Condensação do Quark e Quebra da Sime- tria Eletrofraca}

No capítulo 2 vimos o mecanismo de quebra espontânea de simetria no Modelo Padrão (mecanismo de Higgs), mecanismo que atribui a uma partícula elementar, o bóson de Higgs, a origem para a massa de todas as outras partículas do modelo, férmions e bósons de gauge. Vimos também, que a presença dessa partícula cria o problema da hierarquia entre as escalas de Planck e eletrofraca. Esse problema compromete a naturalidade da teoria e torna a origem da massa do Higgs um mistério.

Esse tipo de problema não acontece na QCD $\Lambda_{Q C D} \sim 100 \mathrm{MeV}$, a teoria das interações fortes, já que a origem de $\Lambda_{Q C D}$ em relação à escala de Planck ocorre de maneira natural $\frac{\Lambda_{Q C D}}{M_{P}} \sim 10^{-20}$, sem necessidade de ajuste fino [38]. Essa foi uma das principais motivações para a criação de teorias Technicolor (teorias fortemente acopladas) nos anos 70 e 80 (Weinberg, Fahri, Suskind), em analogia com a QCD guiadas pela quebra da simetria quiral na teoria BCS da supercondutividade. O modelo de Nambu-Jona-Lasinio (NJL) é a aplicação dessas idéias na física de partículas elementares, mostrando a geração de massa pelas interações fortes através da quebra da simetria quiral.

\section{O Modelo de Nambu-Jona-Lasinio}

Para construir o modelo de NJL vamos partir de uma Lagrangiana efetiva que descreve a interação de um vértice com quatro férmions à altas energias,

$$
\mathcal{L}=\bar{\psi}_{L}^{a} i \not \partial \psi_{L}^{a}+\bar{\psi}_{R}^{a} i \not \partial \psi_{R}^{a}+G \overline{\psi_{L}^{a}} \psi_{R}^{a} \overline{\psi_{R}^{b}} \psi_{L}^{b},
$$

onde a e b são índices de cor do grupo de gauge $S U(N)$, e $G=g^{2} / \Lambda^{2}$, sendo que $g$ é a constante de acoplamento e $\Lambda$ a escala à altas energias onde ocorre a interação. O termo de interação da equação (4.1) vem da interação dos férmions com um bóson de gauge de $S U(N)$, e tem a seginte forma 


$$
\frac{1}{2} g^{2} \bar{\psi}_{L}^{a} \gamma_{\mu} t_{a b}^{A} \psi_{L}^{b} \frac{g^{\mu \nu}}{q^{2}-\Lambda^{2}} \bar{\psi}_{R}^{c} \gamma_{\nu} t_{c d}^{A} \psi_{R}^{d}
$$

Com $q^{2} \ll \Lambda^{2}$ e usando a identidade

$$
\left(t^{A}\right)_{a b}\left(t^{A}\right)_{c d}=\frac{1}{2} \delta_{a d} \delta_{b c}-\frac{1}{2 N} \delta_{a b} \delta_{c d}
$$

obtemos

$$
-\frac{g^{2}}{\Lambda^{2}}\left[\bar{\psi}_{L}^{a} \gamma_{\mu} \psi_{L}^{b} \overline{\psi_{R}^{b}} \gamma^{\mu} \psi_{R}^{a}-\frac{1}{N} \bar{\psi}_{L}^{a} \gamma_{\mu} \psi_{L}^{a} \overline{\psi_{R}^{b}} \gamma^{\mu} \psi_{R}^{b}\right]
$$

Aplicando o rearranjo de Fierz nessa expressão,

$$
\bar{\psi}_{L}^{a} \gamma_{\mu} \psi_{L}^{b} \bar{\psi}_{R}^{b} \gamma^{\mu} \psi_{R}^{a}=-2 \bar{\psi}_{L}^{a} \psi_{R}^{a} \bar{\psi}_{R}^{b} \psi_{L}^{b},
$$

encontramos que o vértice de interação de 4-férmions deve ter a forma

$$
\frac{g^{2}}{\Lambda^{2}}\left[\overline{\psi_{L}^{a}} \psi_{R}^{a} \bar{\psi}_{R}^{b} \psi_{L}^{b}-\frac{1}{N} \bar{\psi}_{L}^{a} \psi_{R}^{b} \bar{\psi}_{R}^{b} \psi_{L}^{a}\right]
$$

sendo que o singleto de cor é atrativo e o octeto de cor é repulsivo e suprimido por $1 / N$. Considerando somente o primeiro termo, a Lagrangiana fica

$$
\mathcal{L}=\bar{\psi}_{L} i \not \partial \psi_{L}+\bar{\psi}_{R} i \not \partial \psi_{R}+\frac{g^{2}}{\Lambda^{2}} \bar{\psi}_{L} \psi_{R} \bar{\psi}_{R} \psi_{L}
$$

Introduzindo um campo escalar auxiliar $H$ (dubleto) na Lagrangiana, podemos reescrevê-la como

$$
\mathcal{L}=\bar{\psi}_{L} i \not \partial \psi_{L}+\bar{\psi}_{R} i \not \partial \psi_{R}+g\left(\bar{\psi}_{L} \psi_{R} H+\text { h.c. }\right)-\Lambda^{2} H^{\dagger} H .
$$

É fácil ver que a integração do campo $H\left(H=\frac{g}{\Lambda^{2}} \bar{\psi}_{R} \psi_{L}\right)$ recupera o vértice de quatro férmions. Em uma escala $\mu<\Lambda$ a Lagrangiana efetiva desenvolve termos cinéticos e de auto interação,

$$
\mathcal{L}=\mathcal{L}_{\text {cin. }}+g\left(\bar{\psi}_{L} \psi_{R}+\text { h.c. }\right)+Z_{H} \partial_{\mu} H^{\dagger} \partial^{\mu} H-m_{H}^{2} H^{\dagger} H-\frac{\lambda_{0}}{2}\left(H^{\dagger} H\right)^{2},
$$


sendo que $Z_{H}=\lambda_{0}=0$ quando $\mu=\Lambda$. Vamos agora calcular os parâmetros $m_{H}, \lambda_{0}$ e $Z_{H}$ à um loop. Para $m_{H}$ o cálculo é semelhante ao da equação (3.3), e no limite $\Lambda \gg \mu$, obtemos

$$
m_{H}^{2}=\Lambda^{2}\left(1-\frac{N g^{2}}{8 \pi^{2}}\right)
$$

Para $Z_{H}$ o diagrama é semelhante, mas com um fator de $1 / k^{2}$, logo

$$
\begin{gathered}
Z_{H} \sim-(-i g)^{2} N \int_{\mu}^{\Lambda} \frac{d k}{k^{4}} k^{3}, \\
Z_{H}=\frac{g^{2} N}{(4 \pi)^{2}} \ln \left(\frac{\Lambda^{2}}{\mu^{2}}\right)
\end{gathered}
$$

e para $\lambda_{0}$ temos que

$$
\begin{gathered}
\sim-(-i g)^{4} N_{c} \int_{\mu}^{\Lambda} \frac{d^{4} k}{(2 \pi)^{4}} \frac{1}{k^{8}} \operatorname{Tr}[\not k \not k \not k], \\
\lambda_{0}=\frac{2 g^{4} N}{(4 \pi)^{2}} \ln \left(\frac{\Lambda^{2}}{\mu^{2}}\right) .
\end{gathered}
$$

Voltando para a equação (4.10), temos duas possibilidades: quando $m_{H}^{2}>0$, a teoria permanece não quebrada; mas para $m_{H}^{2}<0$ a simetria é quebrada espontaneamente, e essa condição requer que $\Lambda^{2}\left(1-\frac{N g^{2}}{8 \pi^{2}}\right)<0 \Longrightarrow g^{2}>$ $\frac{8 \pi^{2}}{N}=g_{c}^{2}$, onde $g_{c}$ é o acoplamento crítico. Para $N=3$ (QCD), por exemplo, $g \gtrsim 5$.

Para obter as consequências físicas da teoria precisamos que ela seja renormalizável. Reparametrizando o campo $H, \tilde{H} \equiv Z_{H}^{1 / 2} H$, a Lagrangiana renormalizável do modelo de NJL é escrita como

$$
\mathcal{L}_{R}=\mathcal{L}_{\text {cin }}+\left|\partial_{\mu} \tilde{H}\right|^{2}+\tilde{g}\left(\bar{\psi}_{L} \psi_{R} \tilde{H}+\text { h.c. }\right)-\tilde{m}_{H}^{2} \tilde{H}^{\dagger} \tilde{H}-\frac{\tilde{\lambda}}{2}\left(\tilde{H}^{\dagger} \tilde{H}\right)^{2}
$$

com os acoplamentos renormalizáveis, 


$$
\begin{gathered}
\tilde{g}^{2}=\frac{g^{2}}{Z_{H}}=\frac{16 \pi^{2}}{N \ln \left(\Lambda^{2} / \mu^{2}\right)}, \\
\tilde{m}_{H}^{2}=\frac{m_{H}^{2}}{Z_{H}}, \\
\tilde{\lambda}=\frac{\lambda_{0}}{Z_{H}^{2}}=\frac{32 \pi^{2}}{N \ln \left(\Lambda^{2} / \mu^{2}\right)} .
\end{gathered}
$$

Esses resultados também poderiam ser encontrados pelas equações do grupo de renormalização. Para obter as consequências fenomenológicas do modelo de NJL vamos analisar o potencial do campo escalar dado por

$$
V=\tilde{m}_{H}^{2}|\tilde{H}|^{2}+\frac{\tilde{\lambda}}{2}|\tilde{H}|^{4}-\tilde{g}\left(\bar{\psi}_{L} \psi_{R} \tilde{H}+\text { h.c. }\right)
$$

onde

$$
\tilde{H}=\left(\begin{array}{c}
0 \\
\frac{v+h}{\sqrt{2}}
\end{array}\right) e^{i \pi^{a} t^{a} / v}
$$

Com a condição $m_{H}^{2}<0$, a simetria é quebrada espontaneamente e o Higgs adquire um $\mathrm{VEV}<\tilde{H}>=\left(\begin{array}{c}0 \\ v / \sqrt{2}\end{array}\right)$, logo, encontramos que a massa do Higgs é

$$
\frac{\partial V}{\partial\left(|\tilde{H}|^{2}\right)}=0 \Longrightarrow m_{h}^{2}=\tilde{\lambda} v^{2}
$$

e o férmion que condensa adquire a massa

$$
m_{\psi}=\frac{\tilde{g} v}{\sqrt{2}}
$$

O modelo de NJL também prevê uma relação entre essas massas,

$$
\frac{m_{h}^{2}}{m_{\psi}^{2}}=2 \frac{\tilde{\lambda} v^{2}}{\tilde{g}^{2} v^{2}}=4
$$




$$
m_{h}=2 m_{\psi}
$$

que só é válida na ausência de interações de gauge, caso contrário $m_{h}<2 m_{\psi}$. Outro resultado importante que podemos obter do modelo é a relação de Pagels-Stokar,

$$
\frac{v^{2}}{2}=\frac{m_{\psi}^{2}}{\tilde{g}^{2}}=m_{\psi}^{2} \frac{N}{16 \pi^{2}} \ln \left(\frac{\Lambda^{2}}{\mu^{2}}\right) .
$$

Como um exemplo de aplicação do modelo de NJL vamos considerar a condensação do top. Sendo o Higgs um estado ligado de $\bar{t}$, a interação atrativa de 4-férmions surge na escala $\Lambda$, formando o condensado

$$
<Q_{L}^{3} t_{R}>\neq 0
$$

que quebra espontaneamente a simetria eletrofraca desde que a condição supercrítica seja satisfeita, isto é, $g^{2}>g_{c}^{2}$. Usando a relação de PagelsStokar com $v \simeq 246 \mathrm{GeV}$ e $m_{t} \simeq 175 \mathrm{GeV}$, obtemos que a quebra dinâmica da simetria eletrofraca deve ocorrer na escala

$$
\Lambda \sim 10^{15} \mathrm{GeV}
$$

Isso cria um problema pois como vimos na seção (3.1.1) a massa do Higgs deve ser da ordem de $1 T e V$, então pela equação (4.10),

$$
m_{H}^{2}=\Lambda^{2}\left(1-\frac{g^{2} N}{8 \pi^{2}}\right) \sim \mathcal{O}\left(v_{\text {fraca }}\right),
$$

e como $g_{c}=8 \pi^{2} / N$, precisamos de um grande ajuste fino para a razão

$$
\frac{g^{2}}{g_{c}^{2}}=1+\mathcal{O}\left(v_{\text {fraca }}^{2} / \Lambda^{2}\right) \sim 1: 10^{-30} .
$$

Como $\Lambda$ não pode estar muito acima da escala $T e V$ a condensação do top não é viável como solução do problema da hierarquia. Para reproduzir a escala $\Lambda \sim 1 \mathrm{TeV}$ o top, ou um quark mais pesado, precisaria ter uma massa 
da ordem de

$$
m_{Q} \approx(600-700) G e V,
$$

que ainda está acordo com a previsão do modelo de NJL $m_{h} \leq 2 m_{Q}$, e respeitando o limite teórico imposto para a massa do Higgs $m_{h} \lesssim 1 \mathrm{TeV}$. Essa é uma das motivações para construção de um modelo de quebra da simetria eletrofraca por uma quarta geração de férmions pesados, a ser discutido nas próximas seções [5], e no qual é baseado esse trabalho.

\subsection{O Modelo}

\subsubsection{Acoplamentos de KK e Operadores de Dimensão Maior}

Para a construção de um modelo de quebra da simetria eletrofraca por uma quarta geração de férmions vamos considerar primeiramente uma teoria com dimensões extras compactas num orbifold $S_{1} / Z_{2}$ com a métrica do modelo de Randall-Sundrum,

$$
d s^{2}=e^{-2 k y} \eta^{\mu \nu} d x_{\mu} d x_{\nu}-d y^{2} .
$$

Como visto anteriormente, essa métrica gera duas escalas efetivas: $M_{P}$ e $M_{P} e^{-\pi k R}$, no intervalo $[0, \pi R]$. Em ordem de resolver o problema da hierarquia, gerar a escala eletrofraca na brana localizada em $y=\pi R$, precisamos de $k R \sim 12$.

Além da gravidade (modelo RS) vamos considerar os férmions, incluindo a quarta geração, e os bósons de gauge se propagando no bulk, com o Higgs localizado na brana IR (infravermelha, TeV) para gerar a escala $\Lambda_{T e V} \simeq$ $M_{P} e^{-\pi k R}$. O grupo de simetria do modelo é $S U(3)_{c} \times S U(2)_{R} \times S U(2)_{L} \times$ $U(1)_{X}$, que é quebrada em $S U(2)_{L} \times U(1)_{Y}$ impondo as condições de contorno na brana UV (ultravioleta, Planck) [39]. 


\section{Acoplamento dos férmions do MP com os bósons de KK}

No capítulo 3 também mostramos como resolver o problema da hierarquia fermiônica no modelo RS, localizando os férmions leves (modos zero) próximo à brana UV (ultravioleta, Planck), enquanto os férmions pesados requerem forte ligação com o Higgs e devem ser localizados próximos à brana IR. Podemos escrever a massa dos férmions no bulk como

$$
m_{f}=c k,
$$

onde o parâmetro c determina a localização dos modos zero dos férmions no bulk. A localização dos modos zero da quarta geração na brana $\mathrm{TeV}$ resulta em fortes interações desses com os modos de KK dos bósons de gauge. Podemos calcular o acoplamento dos férmions com os bóson de KK através da expressão

$$
S_{\text {int }}=g_{5} \int d^{4} x \int d y \sqrt{-g} \bar{\Psi}(x, y) \gamma^{M} A_{M}(x, y) \Psi(x, y),
$$

onde $g_{5}$ é o acoplamento de gauge 5-dimensional, $\sqrt{-g}=e^{-4 k y}$ e $\gamma^{M}=E_{M}^{\alpha} \gamma_{\alpha}$ $=\operatorname{diag} .\left(e^{k y}, e^{k y}, e^{k y}, e^{k y}, 1\right) \gamma_{\alpha}$. Expandindo os campos $A_{M}(x, y)$ e $\Psi(x, y)$ em seus modos de KK (3.26),

$$
A_{M}(x, y)=\frac{1}{\sqrt{2 \pi R}} \sum_{n} A_{\mu}^{(n)}(x) \chi_{n}(y)
$$

e

$$
\Psi_{L, R}(x, y)=\frac{1}{\sqrt{2 \pi R}} \sum_{n} \psi_{L, R}^{(n)}(x) e^{2 k y} f_{L, R}^{(n)}(y)
$$

Substituindo na ação (4.26) e integrando em y obtemos a ação efetiva em $4 \mathrm{D}$,

$$
S_{\text {int }}=g^{(n)} \int d^{4} x \bar{\psi}^{(0)}(x) \gamma^{\mu} A_{\mu}(x) \psi^{(0)}(x),
$$

onde 


$$
g^{(n)}=\frac{g}{\pi R} \int_{0}^{\pi R} d y e^{k y} \chi_{n}(y)\left|f_{L, R}^{(0)}(y)\right|^{2}
$$

é o acoplamento efetivo dos bósons de KK com férmions de modo zero e $g=g_{5} / \sqrt{2 \pi R}$ é o acoplamento de gauge 4-dimensional. As funções de onda para os modos de KK dos bósons de gauge são dadas pela expressão (3.31) com $s=2, \alpha=1$,

$$
\chi_{n}(y)=\frac{e^{k y}}{N_{n}}\left[J_{1}\left(\frac{m_{n}}{k} e^{k y}\right)+b_{1}\left(m_{n}\right) Y_{1}\left(\frac{m_{n}}{k} e^{k y}\right)\right]
$$

com

$$
b_{1}\left(m_{n}\right)=-\frac{J_{0}\left(m_{n} / k\right)}{Y_{0}\left(m_{n} / k\right)} \quad e \quad m_{n} \simeq\left(n-\frac{1}{4}\right) \pi k e^{-\pi k R},
$$

no limite $m_{n} \ll k$ e $k R \gg 1$ para campos pares. As funções de onda para os férmions de modo zero foram calculadas no capítulo precedente e são dadas por $(3.41)$

$$
f_{0}^{R, L}(y)=\sqrt{\frac{(1 \pm 2 c) k \pi R}{e^{(1 \pm 2 c) k \pi R}-1}} e^{ \pm c k y}
$$

sendo que os férmions leves são localizados na brana de Planck e seus parâmetros de massa são $c_{L}>1 / 2, c_{R}<-1 / 2$, enquanto os férmions mais pesados, incluindo a quarta geração, são localizados próximos à brana $\mathrm{TeV}$ com $c_{L}<1 / 2, c_{R}>-1 / 2$. Substituindo $\chi_{n}$ e $f_{0}^{R, L}$ na expressão (4.30) encontramos

$$
\begin{aligned}
g^{(n)}= & g\left(\frac{1 \pm 2 c_{L, R}}{e^{\left(1 \pm 2 c_{L, R}\right) \pi k R}-1}\right) \frac{k}{N_{n}} \int_{0}^{\pi R} d y e^{\left(2 \pm 2 c_{L, R}\right) k y} \\
& \times\left[J_{1}\left(\frac{m_{n}}{k} e^{k y}\right)+b_{1}\left(m_{n}\right) Y_{1}\left(\frac{m_{n}}{k} e^{k y}\right)\right] .
\end{aligned}
$$

A figura (4.1) mostra o comportamento de $g^{(n)} / g$ em função do parâmetro $c_{L}$ para $n=1$. Para valores de $c_{L}$ grandes e negativos $g^{(1)} / g$ se aproxima assintoticamente do limite $\simeq \sqrt{2 \pi k R} \simeq 8.4$, onde $k R \simeq 12$, o qual corre- 
sponde aos férmions confinados na brana $\mathrm{TeV}$ e fortemente acoplados com os bósons de KK, com vínculos para as massas dos bósons de gauge da ordem $m_{1} \gtrsim 20 \mathrm{TeV}$. Para $c_{L}>1 / 2$, férmions localizados na brana $\mathrm{UV}$, os acoplamentos vão assintoticamente para o limite $g^{(1)} / g \simeq-0.2$, pois $\chi_{n}$ é constante nessa região. Isso gera vínculos para massas dos bósons de gauge da ordem $m_{1} \gtrsim 2.1\left(g^{(1)} / g\right) T e V\left(m_{1} \simeq 2.5 k e^{-\pi k R}\right)$, desde que todos os férmions tenham massas 5 -dimensionais iguais.

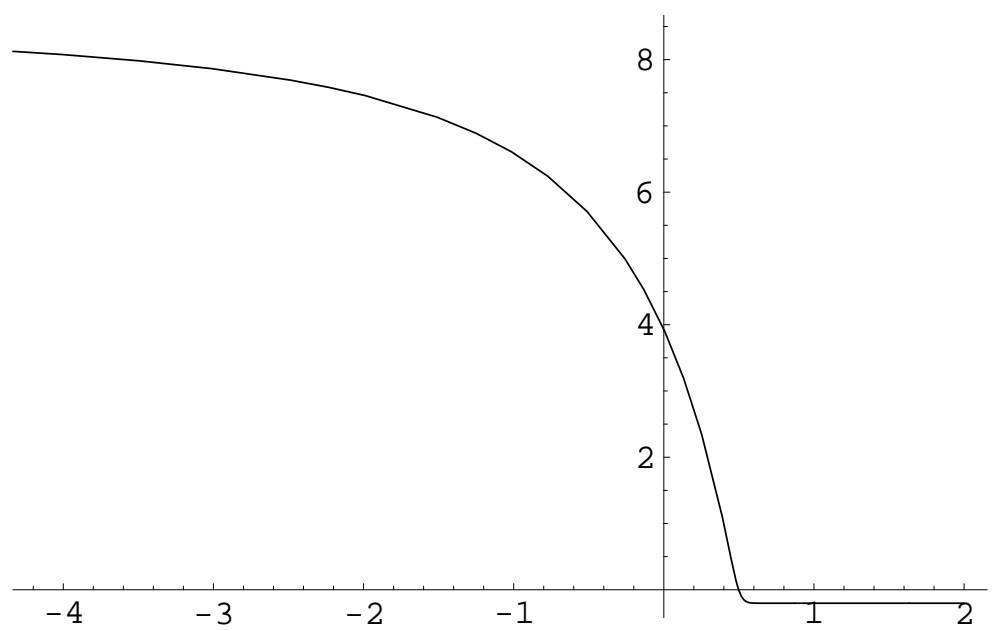

Figura 4.1: Gráfico do acoplamento $g^{(n)} / g$ em função do parâmetro $c_{L}$ para $\mathrm{n}=1$.

\section{Operadores de Dimensão Maior}

Além dos acoplamentos dos férmions do MP e da quarta geração com os bósons de KK, também teremos interações entre quatro férmions induzidas por operadores de dimensão maior no bulk [27, 33], na forma

$$
\int d^{4} x \int d y \sqrt{-g} \frac{C^{i j k l}}{M_{P}^{3}} \bar{\Psi}_{L}^{i}(x, y) \Psi_{R}^{j}(x, y) \bar{\Psi}_{R}^{k}(x, y) \Psi_{L}^{l}(x, y),
$$

onde os coeficientes genéricos 5D $C^{i j k l}$ são estimados na referência [27] por NDA (Naive Dimensional Analysis), 


$$
C^{i j k l} \sim \frac{36 \pi^{3}}{N}
$$

Expandindo os campos fermiônicos $\Psi(x, y)^{\prime} s$ (4.34) com $n=0$ e substituindo na ação de interação para o vértice de 4-férmions, obtemos

$$
\begin{aligned}
& \frac{1}{(\pi R)^{2}} \int d^{4} x \int_{0}^{\pi R} d y e^{-4 k y} \frac{C^{i j k l}}{M_{P}^{3}}(\pi k R)^{2} \\
& \times e^{\left(8-c_{L}^{i}+c_{R}^{j}+c_{R}^{k}-c_{L}^{l}\right) k y} \frac{\bar{\psi}_{L}^{i(0)} \psi_{R}^{j(0)} \bar{\psi}_{R}^{k(0)} \psi_{L}^{l(0)}}{N_{L}^{i} N_{R}^{j} N_{R}^{k} N_{L}^{l}}
\end{aligned}
$$

Integrando na coordenada da dimensão extra e definindo $\tilde{c}_{R}^{i}=-c_{R}^{i}$, encontramos a ação de interação de 4-férmions entre os modos zero dos férmions,

$$
S_{4-f e r .}=\int d^{4} x C^{i j k l} \frac{k}{M_{P}^{3}} \frac{e^{\pi k R\left(4-c_{L}^{i}-\tilde{c}_{R}^{j}-\tilde{c}_{R}^{k}-c_{L}^{l}\right)}-1}{4-c_{L}^{i}-\tilde{c}_{R}^{j}-\tilde{c}_{R}^{k}-c_{L}^{l}} \frac{\bar{\psi}_{L}^{i(0)} \psi_{R}^{j(0)} \bar{\psi}_{R}^{k(0)} \psi_{L}^{l(0)}}{N_{L}^{i} N_{R}^{j} N_{R}^{k} N_{L}^{l}}
$$

que é suprimida exponencialmente quando dois férmions são localizados na brana de Planck $\left(c_{L}^{i, l}, \tilde{c}_{R}^{j, k}>1 / 2\right)$, ou suprimida na escala TeV quando todos os 4-férmions são localizados na brana $\operatorname{IR}\left(c_{L}^{i, l}, \tilde{c}_{R}^{j, k}<1 / 2\right)$. Para um vértice de interação de 4-férmions entre os modos zero da quarta geração, confinados na brana $\mathrm{TeV}$, a constante de acoplamento é dada por

$$
\sim C^{4444}\left(\frac{k}{M_{P}}\right)^{3} \frac{\left(1-2 c_{L}^{4}\right)\left(1-2 \tilde{c}_{R}^{4}\right)}{2\left(2-c_{L}^{4}-\tilde{c}_{R}^{4}\right)}
$$

\subsubsection{Condensação da quarta geração e EWSB}

Na seção anterior calculamos os acoplamentos dos férmions do MP, incluindo a quarta geração, com os bósons de gauge de KK e o acoplamento induzido por operadores de dimensão maior no bulk. Esses acoplamentos são resultados de uma interação de 4-férmions, e em analogia com o modelo de NambuJona-Lasinio podemos escrever uma Lagrangiana de interação de 4-férmions 
com os quarks up da quarta geração na seguinte forma

$$
\mathcal{L}=\bar{U}_{L} i \not D U_{L}+\bar{U}_{R} i \not D U_{R}+\frac{g_{U}^{2}}{M_{K K}^{2}} \bar{U}_{L}^{a} U_{R}^{a} \bar{U}_{R}^{b} U_{L}^{b},
$$

onde a e b são índices de cor $S U(3)_{c}, g_{U}$ são os acoplamentos do quark U da quarta geração, $M_{K K}$ é a massa do primeiro modo de KK do glúon, e a derivada covariante inclui os acoplamentos dos bósons de gauge do grupo $S U(2)_{R} \times S U(2)_{L} \times U(1)_{X}$. O termo de interação pode ser escrito como

$$
-\frac{g_{U}^{2}}{M_{K K}^{2}}\left(\bar{U}_{L} \gamma^{\mu} t^{A} U_{L}\right)\left(\bar{U}_{R} \gamma_{\mu} t^{A} U_{R}\right)
$$

onde $t^{A}$ são os geradores de $S U(3)_{c}$. Usando a identidade (4.3), seguida do rearranjo de Fierz (4.5), obtemos

$$
\frac{g_{U}^{2}}{M_{K K}^{2}}\left[\bar{U}_{L}^{a} U_{R}^{a} \bar{U}_{R}^{b} U_{L}^{b}-\frac{1}{N_{c}} \bar{U}_{L}^{a} U_{R}^{b} \bar{U}_{R}^{b} U_{L}^{a}\right],
$$

onde o acoplamento do quark up da quarta geração tem duas contribuições $(4.33,4.38)$,

$$
g_{U}^{2}=g_{01}^{L} g_{01}^{R}+x_{1}^{2} C_{U U}^{4444}\left(\frac{k}{M_{P}}\right)^{3} \frac{\left(1-2 c_{L}^{4}\right)\left(1-2 \tilde{c}_{R}^{4}\right)}{2\left(2-c_{L}^{4}-\tilde{c}_{R}^{4}\right)},
$$

onde $x_{1} \equiv M_{K K} / \Lambda_{T e V}$ é a massa do primeiro estado excitado do glúon em unidades da escala $T e V, \Lambda_{T e V}=k e^{-\pi k R} \sim \mathcal{O}(1) T e V$, e $g_{01}^{L, R}$ são os acoplamentos dos férmions de modo zero com o primeiro modo de KK do bóson de gauge.

Após o rearranjo de Fierz, a lagrangeana de interação de 4-férmions para o quark U pode ser escrita como

$$
\mathcal{L}=\bar{U}_{L} i \not D U_{L}+\bar{U}_{R} i \not D U_{R}+\frac{g_{U}^{2}}{M_{K K}^{2}} \bar{U}_{L} U_{R} \bar{U}_{R} U_{L},
$$

sendo que não consideramos o octeto de cor suprimido por $1 / N_{c}$. No limite de baixas energias $g_{U}^{2}$ vai atingir um valor supercrítico $\left(g_{U}^{2}>g_{c}\right)$, no qual os quarks vão formar condensados adquirindo um VEV, 


$$
<\bar{U}_{L} U_{R}>\neq 0
$$

quebrando a simetria eletrofraca e gerando uma massa efetiva para o quark U. Para obter a condição crítica para $g_{U}$ vamos partir da Lagrangiana (4.43) introduzindo um campo auxiliar composto, o higgs composto por $\bar{U} U$,

$$
\mathcal{L}=\mathcal{L}_{\text {cin. }}+g_{U}\left(\bar{Q}_{L} H U_{R}+\text { h.c. }\right)-M_{K K}^{2} H^{\dagger} H,
$$

onde $Q_{L}$ é o dubleto de quarks da quarta geração,

$$
Q_{L}=\left(\begin{array}{c}
U \\
D
\end{array}\right)_{L} .
$$

Em uma escala $\mu<M_{K K}$, a Lagrangiana (4.45) desenvolve termos cinéticos e de auto-interação,

$$
\begin{gathered}
\mathcal{L}=Z_{U_{L}} \bar{U}_{L} i \not D U_{L}+Z_{U_{R}} \bar{U}_{R} i \not D U_{R}+Z_{g_{U}} g_{U}\left(\bar{Q}_{L} H U_{R}+\text { h.c. }\right) \\
+Z_{H}\left|D_{\mu} H\right|^{2}-m_{H}^{2} H^{\dagger} H-\frac{\lambda_{0}}{2}\left(H^{\dagger} H\right)^{2},
\end{gathered}
$$

sendo que os parâmetros $Z_{H}, \lambda_{0}$ e $m_{H}$ podem ser calculados a um loop. Esses parâmetros foram calculados no modelo de NJL (4.10 - 4.12) e são dados por

$$
\begin{gathered}
Z_{H}=\frac{g_{U}^{2} N_{c}}{(4 \pi)^{2}} \ln \left(\frac{M_{K K}^{2}}{\mu^{2}}\right), \\
\lambda_{0}=\frac{2 g_{U}^{4} N_{c}}{(4 \pi)^{2}} \ln \left(\frac{M_{K K}^{2}}{\mu^{2}}\right), \\
m_{H}^{2}=M_{K K}^{2}\left(1-\frac{N_{c} g_{U}^{2}}{8 \pi^{2}}\right)+\ldots,
\end{gathered}
$$

onde para $\mu=M_{K K}, \lambda_{0}=0$ e $Z_{H}=0$, definem as chamadas condições de composidade. Podemos agora encontrar o valor supercrítico para $g_{U}$ que 
leva à quebra dinâmica da simetria. A condição para que ocorra a EWSB é $m_{H}^{2}<0, \operatorname{logo}$

$$
g_{U}^{2}>\frac{8 \pi^{2}}{N_{c}}
$$

sendo que para a QCD $\left(N_{c}=3\right), g_{c} \sim 5$, e essa condição é facilmente satisfeita para uma quarta geração de quarks pesados $\left(c_{L}, \tilde{c}_{R}<1 / 2\right)$ interagindo com o primeiro modo de KK do glúon.

Para obter as consequências físicas da teoria precisamos que ela seja renormalizável. Parametrizando os campos do quark U e do Higgs,

$$
Z_{U_{L}}^{1 / 2} U_{L} \rightarrow U_{L}, \quad Z_{U_{R}}^{1 / 2} U_{R} \rightarrow U_{R}, \quad Z_{H}^{1 / 2} H \rightarrow H,
$$

com os acoplamentos renormalizáveis,

$$
\bar{m}_{H}^{2}=\frac{m_{H}^{2}}{Z_{H}}, \quad \bar{\lambda}=\frac{\lambda_{0}}{Z_{H}^{2}}, \quad \bar{g}_{U}=\frac{Z_{g_{U}}}{\sqrt{Z_{U_{L}} Z_{U_{R}} Z_{H}}} g_{U} .
$$

Então a lagrangeana renormalizável pode ser escrita como

$$
\begin{gathered}
\mathcal{L}=\bar{U}_{L} i \not D U_{L}+\bar{U}_{R} i \not D U_{R}+\ldots+\bar{g}_{U}\left(\bar{U}_{L} U_{R} H+\text { h.c. }\right) \\
+\left|D_{\mu} H\right|^{2}-\bar{m}_{H}^{2} H^{\dagger} H-\frac{\bar{\lambda}}{2}\left(H^{\dagger} H\right)^{2}
\end{gathered}
$$

com o potencial do Higgs dado por

$$
V=\bar{m}_{H}^{2} H^{\dagger} H-\frac{\bar{\lambda}}{2}\left(H^{\dagger} H\right)^{2} .
$$

Quando $g_{U}^{2}>g_{c}^{2}$ a simetria é quebrada espontaneamete e o Higgs adquire o VEV

$$
<H>=\left(\begin{array}{c}
0 \\
\frac{v}{\sqrt{2}}
\end{array}\right)
$$


dando massa efetiva para o quark U,

$$
m_{U}=\frac{\bar{g}_{U} v}{\sqrt{2}}
$$

onde $v \simeq 246 \mathrm{GeV}$. A massa do Higgs é $m_{h}=\sqrt{\bar{\lambda}} v$, e o modelo de NJL prêve uma relação entre essas massas que, na ausência de interações de gauge, é dada por

$$
m_{h}=2 m_{U}
$$

Outra previsão importante desse modelo é a relação de Pagels-Stokar (4.20), que relaciona a massa do férmion que condensa com a escala da quebra da simetria eletrofraca $M_{K K}$,

$$
v^{2}=m_{U}^{2} \frac{N_{c}}{8 \pi^{2}} \ln \left(\frac{M_{K K}^{2}}{m_{U}^{2}}\right),
$$

sendo que a EWSB ocorre com a condensação de um quark pesado com massa da ordem $m_{U} \simeq 600 \mathrm{GeV} \Rightarrow M_{K K} \sim \mathcal{O}(1) \mathrm{TeV}$. As previsões para as massas do quark U e do Higgs podem ser melhoradas usando as equações do grupo de renormalização. Esses resultados são calculados na referência [35] e extendidos para a quarta geração em [5], obtendo

$$
\begin{gathered}
m_{U} \sim(600-700) \mathrm{GeV}, \\
m_{h} \sim 1 \mathrm{TeV},
\end{gathered}
$$

que estão em pleno acordo com o modelo de NJL, que prevê $m_{h} \leq 2 m_{U}$ (incluindo as correções ao NJL).

\section{Massa dos férmions}

Como vimos, a condensação dos modos zero dos quarks $U_{R}$ e $U_{L}$ resulta em uma massa dinâmica para esse quark. As massas de todos os outros férmions vêm dos operadores de dimensão maior. Para os férmions leves 
$\left(c_{L}^{i}, \tilde{c}_{R}^{j}>1 / 2\right)$, considerando que $<\bar{U}_{R} U_{L}>\sim m_{U}^{3}$ e usando a expressão (4.34) com $k=l=4$, encontramos

$$
\begin{gathered}
m_{i j}=C^{i j 44}\left(\frac{k}{M_{P}}\right)^{3}\left(\frac{m_{U}}{\Lambda_{T e V}}\right)^{2} \frac{\sqrt{\left(2 c_{L}^{i}-1\right)\left(2 \tilde{c}_{R}^{j}-1\right)} \sqrt{\left(1-2 c_{L}^{4}\right)\left(1-2 \tilde{c}_{R}^{4}\right)}}{4-c_{L}^{i}-\tilde{c}_{R}^{j}-\tilde{c}_{R}^{4}-c_{L}^{4}} \\
\times e^{\pi k R\left(1-c_{L}^{i}-\tilde{c}_{R}^{j}\right)} m_{U}
\end{gathered}
$$

e para os férmions pesados, localizados na brana $\operatorname{IR}\left(c_{L}^{i}, \tilde{c}_{R}^{j}>1 / 2\right)$, obtemos

$m_{i j}=C^{i j 44}\left(\frac{k}{M_{P}}\right)^{3}\left(\frac{m_{U}}{\Lambda_{T e V}}\right)^{2} \frac{\sqrt{\left(1-2 c_{L}^{i}\right)\left(1-2 \tilde{c}_{R}^{j}\right)} \sqrt{\left(1-2 c_{L}^{4}\right)\left(1-2 \tilde{c}_{R}^{4}\right)}}{4-c_{L}^{i}-\tilde{c}_{R}^{j}-\tilde{c}_{R}^{4}-c_{L}^{4}} m_{U}$.

Esse é o caso para o quark top e toda a quarta geração (quark D e léptons).

\subsubsection{Vínculos de precisão eletrofracos}

Vamos agora falar sobre as previsões desse modelo para os parâmetros de Peskin-Takeuchi, que parametrizam potenciais contribuições da nova física às correções radiativas eletrofracas. Esses parâmetros são definidos como [40]:

$$
\begin{gathered}
\alpha S \equiv 4 e^{2}\left[\Pi_{33}^{\prime}(0)-\Pi_{3 Q}^{\prime}(0)\right] \\
\alpha T \equiv \frac{e^{2}}{\operatorname{sen}^{2} \theta_{W} \cos ^{2} \theta_{W} m_{Z}^{2}}\left[\Pi_{11}^{\prime}(0)-\Pi_{33}(0)\right], \\
\alpha U \equiv 4 e^{2}\left[\Pi_{11}^{\prime}(0)-\Pi_{33}^{\prime}(0)\right] .
\end{gathered}
$$

No nosso modelo o grupo de gauge é $S U(3)_{c} \times S U(2)_{L} \times S U(2)_{R} \times U(1)_{X}$, com grande simetria de isospin, que proíbe contribuições do parâmetro $\mathrm{T}$ 
à nível árvore ${ }^{1}$. As contribuições do parâmetro $\mathrm{S}$ à nível árvore para esse modelo de RS no bulk é dada por [39]

$$
S_{A r v .} \simeq 12 \pi \frac{v^{2}}{M_{K K}^{2}}
$$

Com $M_{K K}>2.5 \mathrm{TeV}$, esse parâmetro entra em conflito com a previsão da teoria dual $4 \mathrm{D}$, onde [3]

$$
S_{A r v .} \sim \frac{N}{4 \pi},
$$

sendo $\mathrm{N}$ o tamanho do grupo de gauge da teoria 4D. Uma forma de resolver esse problema seria deslocalizar os férmions leves da brana UV, ao custo de não resolver o problema da massa dos férmions.

Nesse modelo a presença dos férmions da quarta geração induzem novas contribuições a um loop. Nesse caso, o parâmetro S (no limite $m_{U, D}, m_{E, N} \gg$ $m_{Z}$ ) pode ser escrito como

$$
S=\frac{N_{c}}{6 \pi}\left[1-Y \ln \left(\frac{m_{U, E}^{2}}{m_{D, N}^{2}}\right)\right] .
$$

onde $Y$ é a hipercarga dos férmions. Assumindo que $\triangle m \equiv\left|m_{U, E}-m_{D, N}\right| \ll$ $m_{U, D}, m_{E, N}, N_{c}=3$ para quarks e $N_{c}=1$ para os léptons, obtemos

$$
S_{4 G} \simeq \frac{2}{3 \pi}
$$

que não está em contradição com os dados observados [41, 42].

A presença de um Higgs pesado $\left(m_{h} \sim 1 \mathrm{TeV}\right)$ no modelo resulta em uma contribuição positiva para o parâmetro $\mathrm{S}$,

$$
S \simeq \frac{1}{12 \pi} \ln \left[\frac{m_{h}^{2}}{m_{h, r e f .}^{2}}\right],
$$

sendo $m_{h, \text { ref. }}=114 \mathrm{GeV}$, encontramos $S \simeq 0.1$ para $m_{h} \simeq 1 \mathrm{TeV} . \mathrm{Na}$

\footnotetext{
${ }^{1} \mathrm{O}$ parâmetro $\mathrm{T}$ mede a violação de isospin.
} 
referência [43] foi mostrado que nos modelos RS (com ou sem quarta geração) o parâmetro S é divergente a um loop, e portanto deve ser renormalizado e não pode ser usado para vincular o modelo.

Outras contribuições à nível árvore são apresentadas na referência [39]. As contribuições dos férmions de KK a S e T ainda não foram calculadas.

\subsection{Fenomenologia}

A produção de uma quarta geração de férmions [44] tem como consequência uma rica fenomenologia no LHC, com seções de choque da ordem de $1 \mathrm{pb}\left(10^{-36} \mathrm{~cm}^{2}\right)$ para quarks com massas próximas de $\sim 600 \mathrm{GeV}$ [45]. No entanto, a possibilidade de uma quarta geração associada com a quebra da simetria eletrofraca dependerá da descoberta de um Higgs pesado, mas principalmente da observação dos acoplamentos com os bósons de KK [5].

\subsubsection{Quarks da Quarta Geração}

Para os quarks, com $m_{U}>m_{D}$, considerando que somente o quark U condensa, temos dois casos. Se $\left(m_{U}-m_{D}\right)>m_{W}$, então $U \rightarrow W D$ e $D \rightarrow W t$, sendo que para a produção de pares $U \bar{U}$ teremos

$$
U \bar{U} \rightarrow W^{+} W^{-} W^{+} W^{-} W^{+} W^{-} b \bar{b},
$$

sinal que ainda não tem sido estudado no LHC, devido a grande quantidade de jatos. Outra possibilidade seria a produção de $D \bar{D}$,

$$
D \bar{D} \rightarrow W^{+} W^{-} W^{+} W^{-} b \bar{b},
$$

que tem sido estudada na referência [45]. A fenomenologia para a produção de pares $D \bar{D}$ do modelo de EWSB por uma quarta geração, foi estudada na referência [46]. Nesse caso foi analisada a produção de $D \bar{D}$ através da troca de um glúon e do primeiro estado excitado de KK do glúon. Embora esse 
sinal possa ser observado em luminosidades da ordem de $1 \mathrm{fb}^{-1}$, a presença do primeiro modo de KK do glúon será muito difícil de detectar em luminosidades menores que $100 \mathrm{fb}^{-1}$, sendo que essas resonâncias têm larguras entre $\Gamma_{G(1)} \sim(0,7-1,0) M_{G(1)}$, seria muito difícil distingui-las de um sinal com a troca de um glúon da QCD. Uma forma de evitar esse problema seria estudar processos eletrofracos, com os bóson de KK decaindo em léptons da quarta geração, já que essas resonâncias têm larguras estimadas em $(10-20) \%$ de suas massas. Essa é a principal motivação do nosso trabalho, que é estudar a fenomenologia da produção de pares de léptons carregados $E \bar{E}$ e neutrinos $N \bar{N}$ da quarta geração.

\subsubsection{Léptons da Quarta Geração}

Nessa seção apresentamos nosso trabalho, que é estudar a fenomenologia para a produção dos léptons da quarta geração [6]. As seções de choque para a produção dos léptons da quarta geração são muito menores que para os quarks, da ordem de poucos $f b$. Para a produção de pares temos duas possibilidades: se $\left|m_{E}-m_{N}\right|>m_{W}$, então o lépton carregado ou neutrino da quarta geração poderá decair em $(E, N) \rightarrow(N, E) W$; se $\left|m_{E}-m_{N}\right|<m_{W}$, então $(E, N) \rightarrow(\nu, l) W$, podendo ter sinais com grande energia transversa perdida (MET). A primeira possibilidade pode ser excluída considerando que os vínculos eletrofracos ao parâmetro $\mathrm{T}$ impõem que $m_{E} \simeq m_{N}$ [42]. Outra possibilidade seria a produção desses léptons através das correntes carregadas, com $\left(W, W^{(1)}, W_{R}^{(1)}\right) \rightarrow N E$.

\section{Produção de Léptons Carregados}

Nessa seção iremos nos concentrar na produção de pares de léptons carregados da quarta geração. A produção de $E \bar{E}$ no nosso modelo ocorre no LHC da seguinte forma,

$$
p p \rightarrow Z, Z^{(1)}, Z^{\prime(1)}, \gamma, \gamma^{(1)} \rightarrow E \bar{E}
$$


onde $Z^{(1)}$ e $\gamma^{(1)}$ são os estados excitados de KK do $Z$ e do fóton, e o $Z^{\prime}$ é o estado ortogonal do $Z$ associado ao grupo de gauge $S U(2)_{R}$. Uma das vantagens de se estudar esse sinal é que ele tem grande MET - consequentemente vetando backgrounds com pouca MeT - decorrente do decaimento do lépton $E$ em neutrinos das outras três gerações,

$$
E \bar{E} \rightarrow W^{+} W^{-} \nu \bar{\nu}
$$

onde estamos considerando $m_{E} \simeq m_{N}=300 \mathrm{GeV}$, para evitar contribuições ao parâmetro T. Por fim podemos escolher como decair os $W^{\prime} s$. Uma escolha conveniente seria decair um $\mathrm{W}$ em jatos $\left(W^{-}\right)$e o outro em léptons $\left(W^{+} \rightarrow e^{+}, \mu^{+}\right)$. Para esse sinal temos os seguintes backgrounds:

i) $W^{+} j j$, com o $W^{+}$decaindo em léptons;

ii) $W^{+} W^{-} Z$, com o $W^{+}$decaindo em léptons, $W^{-}$decaindo em jatos e o $Z$ decaindo em neutrinos;

iii) $t \bar{t}$, com $t \rightarrow W b$ e os $W^{\prime} s$ decaindo em léptons, sendo um lépton perdido no detector.

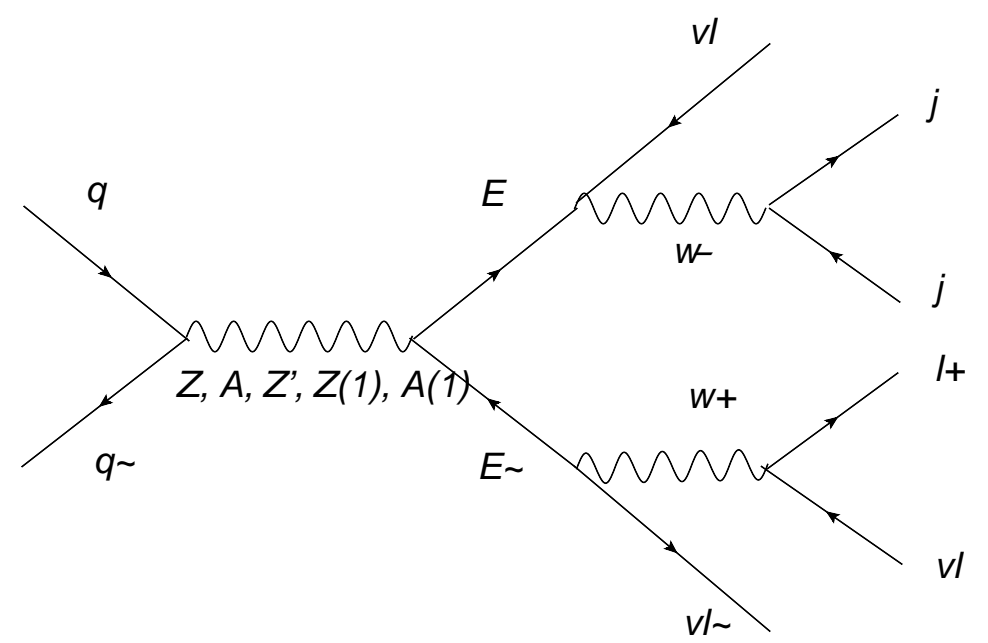

Figura 4.2. Diagrama para o sinal (4.70). 


\section{Produção de Neutrinos}

Um outro sinal importante para a observação dos bósons de KK seria através da produção de pares de neutrinos da quarta geração. A produção de $\bar{N} N$ é semelhante ao caso dos léptons carregados, mas sem acoplamentos com o fóton,

$$
p p \rightarrow Z, Z^{(1)}, Z^{\prime(1)} \rightarrow \bar{N} N
$$

com os neutrinos decaindo em

$$
\bar{N} N \rightarrow W^{+} W^{-} e^{-} \mu^{+},
$$

com $m_{E} \simeq m_{N}$, sem considerar o decaimento em $\tau$. Nesse caso, vamos decair ambos os $W^{\prime} s$ em jatos, vetando qualquer fundo com muita MET. Alguns backgrounds possíveis para esse caso são:

i) $\bar{t} t j j$, com $t \rightarrow W b$, e os $W^{\prime} s$ decaindo leptonicamente;

ii) $W^{+} W^{-} j j j j$, com os $W^{\prime} s$ decaindo leptonicamente.

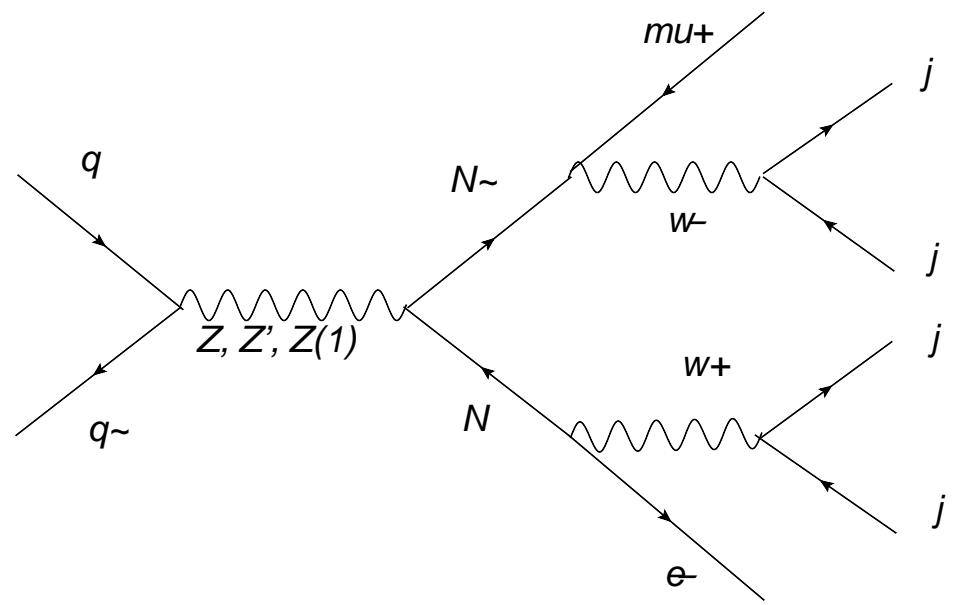

Figura 4.3. Diagrama para o sinal (4.72).

\section{Larguras e acoplamentos}

Antes de prosseguirmos com a análise dos sinais, precisamos especificar os acoplamentos e larguras que estamos usando no nosso modelo. 
Os acoplamentos dos férmions da quarta geração com os bósons de gauge $Z, W^{ \pm}, \gamma$, são os mesmos do Modelo Padrão, mas para o decaimento dos $W^{\prime} s$ em léptons precisamos adicionar o elemento de matriz PMNS $V_{i j}$, relacionado com a mistura entre diferentes gerações. Em particular, para a mistura de um lépton da quarta geração com as outras gerações $(i=e, \mu, \tau)$ temos que

$$
V_{4 i} \lesssim 0.01
$$

que foi estimado considerando o limite imposto pelo decaimento $\mu^{-} \rightarrow e^{-} \gamma$ $2[?]$.

Os acoplamentos dos férmions do MP e da quarta geração com os bósons de KK foram calculados na seção (4.2.1). A equação (4.33) mostra que esse acoplamento $g^{(n)}$ depende do fator de localização c, que está relacionado com as massas dos férmions de acordo com as expressões (4.59 e 4.60). Sendo assim, os parâmetros $c_{L}$ e $c_{R}$ podem ser ajustados de acordo com as massas da quarta geração, e os valores possíveis para esses parâmetros são $c_{L}<1 / 2, c_{R}>-1 / 2$ (para férmions pesados, localizados na brana IR). Considerando somente a produção de pares $N \bar{N}$ e $E \bar{E}$ de mão esquerda, podemos escolher

$$
c_{L}=0 \text {. }
$$

Nesse caso, o acoplamento dos léptons da quarta geração com o primeiro estado excitado dos bósons de KK é $g^{(1)} \simeq 3.9 g$, onde $g$ é o acoplamento do MP.

Os acoplamentos dos léptons da quarta geração com o primeiro modo de KK do $Z^{\prime}\left(Z^{\prime}\right.$ não tem modo zero) é dado por

$$
g_{Z^{\prime}}^{(1)}\left(L_{L}\right)=3.9 \times \frac{1}{2} \frac{g_{X}^{2}}{\sqrt{g^{2}+g_{X}^{2}}}
$$

onde $g_{X}$ pode ser obtido da relação $g^{\prime}=g_{R} g_{X} / \sqrt{g_{R}^{2}+g_{X}^{2}}$, escolhendo $g_{R}=$

\footnotetext{
${ }^{2}$ Muito suprimido no MP.
} 
$g_{L}=g$. Os acoplamentos do $Z^{\prime}$ com os outros férmions do MP e da quarta geração são dados por

$$
\begin{gathered}
g_{Z^{\prime}}\left(Q_{L}\right)=\frac{\left(-1 / 2 g^{2}-2 / 3 g_{X}^{2}\right)}{\sqrt{g^{2}+g_{X}^{2}}} \times f l, \quad g_{Z^{\prime}}\left(u_{R}\right)=-\frac{2}{3} \frac{g_{X}^{2}}{\sqrt{g^{2}+g_{X}^{2}}} \times f l \\
g_{Z^{\prime}}\left(d_{R}\right)=\frac{1}{3} \frac{g_{X}^{2}}{\sqrt{g^{2}+g_{X}^{2}}} \times f l \\
g_{Z^{\prime}}\left(l_{R}\right)=-\frac{1}{2} \frac{g_{X}^{2}-g^{2}}{\sqrt{g^{2}+g_{X}^{2}}} \times f l, \quad g_{Z^{\prime}}\left(v_{R}\right)=\frac{1}{2} \sqrt{g^{2}+g_{X}^{2}} \times f l
\end{gathered}
$$

onde $f l$ é o fator de localização dado pela relação $g^{(n)} / g$ (nesse caso $g=g_{Z^{\prime}}$ ). Todos os acoplamentos entre os primeiros modos de KK dos bósons de gauge e os férmions são ilustrados na tabela 4.1.

\begin{tabular}{|c|c|c|c|c|c|c|c|c|c|}
\hline Bósons KK & $q_{i}, l_{i}$ & $q_{L}^{3}$ & $t_{R}$ & $Q_{L}^{4}$ & $U_{4 R}$ & $D_{4 R}$ & $L_{L}^{4}$ & $E_{4 R}$ & $N_{4 R}$ \\
\hline \hline$Z^{(1)}, Z^{\prime(1)}$ & -0.2 & 0.6 & 3.9 & 3.7 & 4.4 & 2.8 & 3.9 & 5.42 & $5.13,\left(Z^{\prime(1)}\right)$ \\
\hline$\gamma^{(1)}$ & -0.2 & 0.6 & 3.9 & 3.7 & 4.4 & 2.8 & $3.9,\left(E_{L}\right)$ & 5.42 & - \\
\hline
\end{tabular}

Tabela 4.1. Fatores de localização $g^{(n)} / g$, onde $g$ é o acoplamento do MP para $Z^{(1)}$ e $\gamma^{(1)}$, ou os acoplamentos (4.54) para o $Z^{\prime(1)}$.

Conhecendo os acoplamentos podemos calcular as larguras dos bósons de $\mathrm{KK} Z^{(1)}, Z^{\prime(1)}$ e $\gamma^{(1)}$, e dos férmions da quarta geração. Calculamos essas larguras analiticamente e usando o programa BRIDGE. Para os léptons da quarta geração encontramos

$$
\Gamma_{E, N}=\frac{G_{F} m_{E, N}^{3}}{8 \pi \sqrt{2}}\left|V_{i j}\right|^{2},
$$

Considerando somente o decaimento nas outras 3 gerações com $m_{E}=m_{N}=$ $300 \mathrm{GeV}$, obtemos

$$
\Gamma_{E, N} \simeq 2.65 \mathrm{MeV}
$$


As larguras para os estados excitados do fóton, $Z^{(1)}$ e $Z^{\prime(1)}$ decaindo em férmions, calculadas analiticamente, são dadas por

$$
\begin{gathered}
\Gamma_{\gamma(1)} \simeq \frac{M_{\gamma(1)} \operatorname{sen}^{2} \theta_{W}}{12 \pi}\left(g_{L}+g_{R}\right)^{2}, \\
\Gamma_{Z_{1}, Z_{1}^{\prime}} \simeq N_{c} \frac{4 G_{F} m_{Z}^{2}}{3 \pi \sqrt{2}} M_{Z_{1}, Z_{1}^{\prime}}\left(g_{L}^{2}+g_{R}^{2}\right) .
\end{gathered}
$$

Considerando os acoplamentos com os férmions e os decaimentos bosônicos obtemos

$$
\begin{aligned}
& \Gamma_{\gamma(1)} \simeq 360 \mathrm{GeV}, \\
& \Gamma_{Z(1)} \simeq 630 \mathrm{GeV}, \\
& \Gamma_{Z^{\prime}(1)} \simeq 870 \mathrm{GeV},
\end{aligned}
$$

onde usamos $M_{K K}=2.4 \mathrm{TeV}$ para as massas dos bósons de KK.

\section{Seções de Choque}

Com todos os acoplamentos e larguras, finalmente calculamos as seções de choque para a produção dos léptons da quarta geração, utilizando o programa gerador de eventos MadGraph/MadEvent, obtendo

$$
\begin{gathered}
\sigma_{E \bar{E}} \simeq 31 \mathrm{fb}, \\
\sigma_{\bar{N} N} \simeq 25 \mathrm{fb} .
\end{gathered}
$$

Para $E \rightarrow W \nu$ não temos nenhuma perda na seção de choque, pois o lépton carregado da quarta geração sempre vai decair nas outras três gerações. 
Com o $W^{-}$decaindo em jatos e o $W^{+} \rightarrow l^{+} \nu\left(l^{+}=e^{+}, \mu^{+}\right)$, temos as BR's (Branching Ratios) 2/3, 2/9 respectivamente, logo

$$
\sigma_{\text {sin. }(\bar{E} E)} \simeq \frac{2}{3} \times \frac{2}{9} \times \sigma_{\bar{E} E} \simeq 4.59 \mathrm{fb} .
$$

Para os neutrinos da quarta geração com $N \rightarrow W l\left(l=e^{-}, \mu^{+}\right)$temos uma BR de $2 / 9$, e com os decaimentos dos W's em jatos $4 / 9$, logo

$$
\sigma_{\text {sin. }(\bar{N} N)} \simeq \frac{2}{9} \times \frac{4}{9} \times \sigma_{\bar{N} N} \simeq 2.47 \mathrm{fb} .
$$

Uma análise preliminar para a produção de $\bar{E} E$ impondo os cortes mínimos do experimento

$$
\begin{gathered}
p_{T}^{j}>20 \mathrm{GeV}, \quad\left|\eta_{j}\right|<3.0, \\
p_{T}^{l}>10 \mathrm{GeV}, \quad\left|\eta_{l}\right|<2.5, \\
\triangle R_{j j} \geq 0.7 \quad, \quad \Delta R_{l j} \geq 0.7, \quad \triangle R_{l l} \geq 0.7,
\end{gathered}
$$

reduz a seção de choque para $\sigma_{\sin .(\bar{E} E)} \simeq 2.31 f b$. Na equação $(4.81) p_{T}$ é a componente do momento no plano transversal, $\eta$ é a chamada pseudorapidity, definida como $\eta=-\ln \left(\tan \left(\theta_{C M}\right) / 2\right)$, onde $\theta_{C M}$ é o ângulo de espalhamento do centro de massa, e $\triangle R$ é a separação angular entre as partículas do mesmo feixe. A imposição de outros cortes para eliminação do background, por exemplo, para a MET e o $p_{T}^{l}$ reduz ainda mais as seções de choque para o sinal $\bar{E} E$. No caso dos neutrinos da quarta geração temos observado que os cortes podem não ser tão grandes, tornando a observação do sinal mais fácil que no caso do lépton carregado, com seções de choque da ordem de $1 \mathrm{fb}$. Outra possibilidade que estamos estudando é a produção dos léptons da quarta geração através de correntes carregadas $W, W^{(1)}, W_{R}^{(1)} \rightarrow E, N$, com seções de choque de produção da ordem de $40 \mathrm{fb}$, é possível que as seções de choque para esse sinal após os decaimentos e cortes sejam maiores 
que nos casos $\bar{E} E$ e $\bar{N} N$. Como consequência a observação de sinais para a produção de léptons da quarta geração no LHC deve exigir luminosidades com dezenas $f b^{-1}$. 


\section{Capítulo 5}

\section{Conclusão}

Iniciamos esse trabalho apresentando o Modelo Padrão da física de partículas elementares. Começamos pelos ingredientes básicos da teoria quântica de campos necessários para a construção do modelo, a invariância de gauge, a quebra espontânea de simetria e o mecanismo de Higgs. Em seguida estudamos o modelo das interações eletrofracas, a Lagrangiana do modelo, as correntes carregadas e neutras, o mecanismo de quebra espontânea de simetria do modelo e suas previsões. Nas últimas seções desse capítulo introduzimos os quarks, a teoria das interações fortes (QCD), as anomalias do modelo GWS, a matriz CKM e a Lagrangiana total do MP, incluindo todas as interações.

No capítulo seguinte discutimos a física além do MP. Começamos abordando os principais problemas do MP, o problema da hierarquia e o problema da massa dos férmions. Comentamos brevemente outros problemas do modelo, o problema da constante cosmológica, a matéria escura, o problema da violação de CP nas interações fortes e a assimetria matéria-antimatéria. Na última seção introduzimos as principais teorias físicas além do modelo padrão, nas quais é baseado o nosso trabalho, as teorias com dimensões extras curvas (modelo de Randall-Sundrum).

Iniciamos o último capítulo apresentando um modelo de quebra da simetria eletrofraca, o modelo de Nambu-Jona-Lasinio, ferramenta necessária para a 
construção do modelo que estamos estudando [5]. Como vimos, esse modelo propõe uma quarta geração de férmions fortemente acoplada na escala $\mathrm{TeV}$ como origem da quebra da simetria eletrofraca. Temos então uma teoria 4D fortemente acoplada que pode ser descrita por uma teoria dual 5D fracamente acoplada de acordo com a correspondência AdS/CFT. Como consequência os acoplamentos desse modelo têm duas contribuições, as interações dos férmions do MP e da quarta geração com os bósons de KK, e as interações induzidas por operadores de dimensão maior. Vimos que a primeira contribuição é fundamental para gerar a condição supercrítica no modelo de NJL $g>g_{c}$, necessária para que os quarks U da quarta geração condensem e quebrem a simetria eletrofraca. A segunda contribuição por sua vez, gera massa para todos os outros férmions do modelo, resolvendo o problema da massa dos férmions.

Na última seção do capítulo 4 estudamos a fenomenologia desse modelo para a produção de quarks e léptons. A fenomenologia para a produção de quarks do modelo foi estudada na referência [46]. Nosso trabalho se concentrou na análise da produção de léptons do modelo, mais especificamente em processos envolvendo a produção de pares de léptons carregados e neutrinos da quarta geração [6]. Antes de calcularmos as seções de choque para a produção dos nossos sinais especificamos todos os acoplamentos e larguras do modelo. Para a produção de $\bar{E} E$, com $E \rightarrow W v$, temos um sinal com muita energia transversa perdida (MET), e isso nos permite eliminar grande parte do background quando pedimos um corte para grandes valores de MET, da ordem de $300 \mathrm{GeV}$ para um sinal com $5 \sigma$ de significância. Para a produção de $\bar{N} N, \operatorname{com} N \rightarrow W l\left(l=e^{-}, \mu^{-}\right)$e os $W^{\prime} s$ decaindo em jatos, temos o caso contrário, um sinal sem MET, e portanto podemos eliminar backgrounds com muita MET.

Argumentamos que a motivação principal para se estudar a produção de léptons da quarta geração é a possibilidade de observarmos os bósons de KK, já que as larguras para esses bósons devem ser da ordem de 20\% das suas massas, muito menor que na produção via QCD onde $\Gamma_{G(1)} \simeq(0.7-1.0) M_{G(1)}$. Pretendemos dar continuidade a esse trabalho estudando todos os backgrounds 
possíveis para a produção dos léptons da quarta geração, em seguida melhorando os cortes para obter um sinal mais claro. Sendo assim, o objetivo também é estudar sinais envolvendo correntes carregadas, $W, W^{(1)}, W_{R}^{(1)} \rightarrow E, N$, processos com seções de choque da ordem de $\sim 40 \mathrm{fb}$. A idéia é que, embora os processos eletrofracos tenham baixas seções de choque de produção em relação aos quarks, eles são o melhor caminho para a observação dos bósons de KK acoplados com a quarta geração, corroborando o modelo que estamos estudando. 


\section{Referências Bibliográficas}

[1] S. L. GLASHOW, Partial-Symmetries of weak interactions, Nucl. Phys., 22, 579 (1961).

[2] S. WEINBERG, A model of leptons, Phys. Rev. Lett., 19, 1264 (1967).

[3] G. BURDMAN, Holographic Models of Electroweak Symmetry Breaking, arXiv:hep-ph/0806.4000 (2008).

[4] J. MALDACENA, The large $N$ limit of superconformal field theories and supergravity, Adv. Theor. Math. Phys., 2 (1998), 231.

[5] G. BURDMAN AND L. DA ROLD, Electroweak Symmetry Breaking from a Holographic Fourth Generation, JHEP, 0712, 086 (2007).

[6] G. BURdman, L. DA ROLD, O. J. P. ÉBOli, C. E. F. HALUCH, AND R. D. MATHEUS, em andamento (2009).

[7] A. SALAM AND J. C. WARD, Electromagnetic and weak interactions, Phys. Lett., 13, 168 (1964).

[8] C. N. YANG AND R. L. MILLS, Conservation of isotopic spin and isotopic gauge invariance, Phys. Rev., 96, 191 (1954).

[9] J. SCHWINGER, A theory of the fundamental interactions, Ann Phys. (N. Y.), 2, 407 (1957).

[10] S. F. NOVAES, Standard Model: An Introduction, arXiv:hepph/0001283 (2000). 
[11] G. 't HOOFT, Renormalization of massless Yang-Mills fields, Nucl. Phys., B 33, 173 (1971).

[12] G. 't HOOFT, Renormalizable for massive Yang-Mills fields, Nucl. Phys., B 35, 167 (1971).

[13] C. AMSLER ET AL. [PDG], Physics Letters B667, 1 (2008).

[14] S. L. GLASHOW, J. ILIOPOULOS, AND L. MAIANI, Weak interactions with lepton-hadron symmetry, Phys. Rev., D 2, 1285 (1970).

[15] J. F. DONOGHUE, E. GOLOWICH AND B. HOLSTEIN, Dynamics of the Standard Model, Cambridge University Press, 1994.

[16] B. W. LEE, C. QUIGG, AND H. B. THACKER, Weak interactions at very high energies: The role of the Higgs-boson mass, Phys. Rev., D 16, 1519 (1977).

[17] C. QUIGG, Gauge Theories of the Strong, Weak, and Eletromagnetic Interactions, Westview Press, 1983, 1997.

[18] M. E. PESKIN AND D. V. SCHROEDER, An Introduction to Quantum Field Theory, HarperCollins Publishers, 1995.

[19] P. M. DE AQUINO, Física além do Modelo Padrão em Teorias com Dimensões Extras, Dissertação de Mestrado, Universidade de São Paulo (2007).

[20] R. D. PECCEI AND H. R. QUINN, Constraints imposed by CP conservation in the presence of instantons, Phys. Rev., D 16, 1791 (1977).

[21] A. PÉREZ-LORENZANA, An Introduction to Extra Dimensions, arXiv:hep-ph/0503177 (2005).

[22] R. SUNDRUM, To the Fifth Dimension and Back, axXiv:hepth/0508134 (2005). 
[23] N. ARKAni HAMED, S. DimOpoulos, AND G. R. DIVAli, The Hierarchy Problem and New Dimensions at a Millimeter, Phys. Lett., B 429, 263 (1998).

[24] G. BURDMAN, Theories with Extra Dimensions, arXiv:hepph/0409322 (2004).

[25] T. APPelquist, H. C. CHEnG, AND B. A. DOBRESCU, Bounds on Universal Extra Dimensions, Phys.Rev., D 64, 035002 (2001).

[26] L. RANDALL AND R. SUNDRUM, A Large Mass Hierarchy from a Small Extra Dimension, Phys. Rev. Lett., 83, 3370 (1999).

[27] T. GHERGHETTA AND A. POMAROL, Bulk fields and supersymmetry in a slice of AdS, Nucl. Phys., B 586, 141 (2000).

[28] W. D. GOLDBERGER AND M. B. WISE, Bulk fields in the RandallSundrum compactification scenario, Phys. Rev., D 60, 107505 (1999).

[29] H. DAVOUdiAsL, J. L. HEWETT, AND T. G. RIZZO, Bulk Gauge Fields in the Randall-Sundrum Model, Phys. Lett., B 473, 43 (2000).

[30] S. CHANG, J. HISANO, H. NAKANO, N. OKADA, AND M. YAMAGUCHI, Bulk Standard Model in the Randall-Sundrum Background, Phys. Rev., D 62, 084025 (2000).

[31] Y. GROSSMAN AND M. NEUBERT, Neutrino masses and mixings in non-factorizable geometry, Phy. Lett., B 474, 361 (2000).

[32] A. POMAROL, Gauge bosons in a five-dimensional theory with localized gravity, Phys. Lett., B 486, 153 (2000).

[33] S. J. HUBER AND Q. SHAFI, Fermion masses, mixings and proton decay in a Randall-Sundrum model, Phys. Lett., B 498, 256 (2001).

[34] G. BURDMAN, Constraints on the bulk standard model in the RandallSundrum scenario, Phys. Rev., D 66, 076003 (2002). 
[35] W. A. BARDEEN, C. T. HILL, AND M. LINDNER, Minimal dynamical symmetry breaking of the standard model, Phys. Rev. D 41, 1647 (1990).

[36] C. CSÁKI, J. HUBISZ, AND P. MEADE, TASI Lectures on Electroweak Symmetry Breaking from Extra Dimensions, arXiv:hep-ph/0510275 (2005).

[37] T. GHERGHETTA, Les Houches Lectures on Warped Models and Holography, arXiv:hep-ph/0601213 (2006).

[38] C. T. HILL AND E. H. SIMMONS, Strong Dynamics and Electroweak Symmetry Breaking, Phys.Rept. 381, 235 (2003), [Erratium-ibid. 390, 553 (2004)].

[39] K. AGASHE, A. DELGADO, M. J. MAY, AND R. SUNDRUM, RS1, Custodial Isospin and Precision Tests, JHEP, 0308, 050 (2003).

[40] M. E. PESKIN AND T. TAKEUCHI, Estimation of oblique electroweak corrections, Phys. Rev., D 46, 381 (1992).

[41] W. M. YAO AND ET AL. [PDG], Review of particle physics, J. of Phys. G 33 (2006) 1.

[42] G. D. KRIBS, T. PLEHN, M. SPANNOWSKY, AND T. M. P. TAIT, Four Generations and Higgs Physics, Phys.Rev., D 76, 075016 (2007).

[43] G. BURDMAN AND L. DA ROLD, Renormalization of the S Parameter in Holographic Models of Electroweak Symmetry Breaking, JHEP, 0811, 025 (2008).

[44] B. HOLDOM, The discovery of the fourth family at the LHC: what if?, JHEP, 0608, 076 (2006).

[45] ATLAS Detector and Physics Technical Design Report, Volume I, CERN-LHCC-99-14, ATLAS-TDR-14, May 1999.

[46] G. BURDMAn, L. DA ROLD, O. J. P. ÉBOLI, AND R. D. MATHEUS, A Strongly Coupled Fourth Generation at the LHC, Phys. Rev. D 79, 075026 (2009). 